Dynamical computation of constrained flexible systems using a modal Udwadia-Kalaba formulation: Application to musical instruments

J. Antunes and V. Debut

Citation: J. Acoust. Soc. Am. 141, 764 (2017); doi: 10.1121/1.4973534

View online: http://dx.doi.org/10.1121/1.4973534

View Table of Contents: http://asa.scitation.org/toc/jas/141/2

Published by the Acoustical Society of America 


\title{
Dynamical computation of constrained flexible systems using a modal Udwadia-Kalaba formulation: Application to musical instruments
}

\author{
J. Antunes ${ }^{1}$ and V. Debut ${ }^{2, a)}$ \\ ${ }^{1}$ Centro de Ciências e Tecnologias Nucleares, Instituto Superior Técnico, Universidade de Lisboa, \\ Estrada Nacional 10, Km 139.7, Bobadela LRS, 2695-066, Portugal \\ ${ }^{2}$ Instituto de Etnomusicologia-Centro de Estudos em Música e Dança, Faculdade de Ciências Sociais e \\ Humanas, Universidade Nova de Lisboa, Avenida de Berna, 26C, Lisbon, 1069-061, Portugal
}

(Received 15 May 2016; revised 23 November 2016; accepted 8 December 2016; published online 8 February 2017)

\begin{abstract}
Most musical instruments consist of dynamical subsystems connected at a number of constraining points through which energy flows. For physical sound synthesis, one important difficulty deals with enforcing these coupling constraints. While standard techniques include the use of Lagrange multipliers or penalty methods, in this paper, a different approach is explored, the Udwadia-Kalaba (U-K) formulation, which is rooted on analytical dynamics but avoids the use of Lagrange multipliers. This general and elegant formulation has been nearly exclusively used for conceptual systems of discrete masses or articulated rigid bodies, namely, in robotics. However its natural extension to deal with continuous flexible systems is surprisingly absent from the literature. Here, such a modeling strategy is developed and the potential of combining the U-K equation for constrained systems with the modal description is shown, in particular, to simulate musical instruments. Objectives are twofold: (1) Develop the U-K equation for constrained flexible systems with subsystems modelled through unconstrained modes; and (2) apply this framework to compute string/body coupled dynamics. This example complements previous work [Debut, Antunes, Marques, and Carvalho, Appl. Acoust. 108, 3-18 (2016)] on guitar modeling using penalty methods. Simulations show that the proposed technique provides similar results with a significant improvement in computational efficiency. (C) 2017 Acoustical Society of America.
\end{abstract}

[http://dx.doi.org/10.1121/1.4973534]

[JFL]

Pages: $764-778$

\section{INTRODUCTION}

This paper deals with flexible constrained systems and their effective dynamical modeling and computation in the particular context of physical synthesis of musical instruments. Most musical instruments consist of dynamical subsystems connected at a number of constraining points through which tuning is achieved or the vibratory energy flows. Coupling is therefore an essential feature in musical instruments and, when addressing physically based synthesis, most modeling and computational difficulties are connected with the manner in which the coupling constraints are enforced. Typically, these are modelled using standard techniques such as Lagrange multipliers or penalty methods, each one with specific merits and drawbacks. In this paper we explore a different approach-the Udwadia-Kalaba (U-K) formulation, originally proposed in the early 1990s for discrete constrained systems; see Udwadia and Kalaba $(1992,1996)$ - which is anchored on analytical dynamics but avoids the use of Lagrange multipliers. In particular, leading to constrained formulations in terms of standard ordinary differential equation (ODE) systems, even when a redundant set of coordinates is used, instead of numerically challenging mixed differential-algebraic equations (DAEs).

\footnotetext{
${ }^{\text {a)} E l e c t r o n i c ~ m a i l: ~ v i n c e n t d e b u t @ f c s h . u n l . p t ~}$
}

Up to now, this general, very elegant, and appealing formulation has been nearly exclusively used to address conceptual systems of discrete masses or articulated rigid bodies, namely, in robotics. To the authors' best knowledge, the single exception in the literature is the work by Pennestri et al. (2010), who addressed a flexible slider-crank mechanism, modelled using a finite element Timoshenko beam formulation. However, in spite of the possible natural extension of the U-K formulation to deal with flexible systems modelled through their unconstrained modes, such a promising approach is surprisingly absent from the literature. In the present work we develop the potential of combining the U-K formulation for constrained systems with the modal description of flexible structures in order to achieve reliable and efficient computations of dynamical responses, in particular, for simulating the transient responses of musical instruments.

The objectives of this paper are thus twofold: (1) We develop the U-K equation for constrained flexible systems in which the various sub-structures are modelled through unconstrained modal basis; and (2) we apply this formulation to compute the dynamical responses of a guitar string coupled to the instrument body at the bridge. This illustration complements extensive work already performed in the past by the authors on guitar string/modeling using penalty methods; see Marques et al. (2013) and Debut et al. (2014, 2015, 2016), thus, enabling an interesting comparison 
between the computational efficiency of different modeling strategies.

In Sec. II, we briefly recall the essentials behind the U$\mathrm{K}$ formulation. Then, we develop in Sec. III the modal U-K formulation for general flexible constrained systems modelled through the unconstrained modes of the various substructures. In Sec. IV, this theoretical formulation is illustrated by modeling a musical string instrument consisting on two vibrating sub-structures - a guitar string and bodywith several constraints. These are enforced at the instrument bridge where the string and body motions must be identical, as well as at string tuning locations on the fingerboard where string motion is restricted. Finally, in Sec. V, we present illustrative results obtained from the guitar model computed using the modal U-K approach, and present a comparison with results stemming from a penalty-based formulation.

These results demonstrate the computational efficiency of the proposed technique, which for the application at hand achieved simulations of comparable quality with a 2-orderof-magnitude improvement in computational efficiency.

\section{THEORETICAL FORMULATION}

The original U-K formulation was deduced from Gauss' principle of least action. A simple and elegant approach for obtaining the U-K formulation for constrained systems, which may be found in the interesting papers by Arabyan and Wu (1998) and Laulusa and Bauchau (2008), is briefly recalled here for completeness. A system of $M$ particles with mass matrix $\mathbf{M}$ is subjected to an external force vector $\mathbf{F}_{e}(t)$ of constraint-independent forces and a set of $P=P_{h}+P_{n h}$ holonomic and non-holonomic constraints, depending on the system displacement $\mathbf{x}(t)$ and velocity $\dot{\mathbf{x}}(t)$, as well as explicitly on time, given by the general equations

$$
\begin{aligned}
& \varphi_{p}(\mathbf{x}, t)=0, \quad p=1,2, \ldots, P_{h}, \\
& \psi_{p}(\mathbf{x}, \dot{\mathbf{x}}, t)=0, \quad p=P_{h}+1,2, \ldots, P,
\end{aligned}
$$

which, by double or single time-differentiation, may be written as a general matrix-vector constraint system in terms of accelerations

$$
\left[\begin{array}{cc}
\mathbf{M} & \mathbf{A}^{T} \\
\mathbf{A} & \mathbf{0}
\end{array}\right]^{-1}=\left[\begin{array}{c}
\mathbf{M}^{-1}-\underset{\mathbf{M}^{-1} \mathbf{A}^{T}\left(\mathbf{A} \mathbf{M}^{-1} \mathbf{A}^{T}\right)^{-1} \mathbf{A} \mathbf{M}^{-1}}{\left(\mathbf{A M}^{-1} \mathbf{A}^{T}\right)^{-1} \mathbf{A} \mathbf{M}^{-1}}
\end{array}\right.
$$

leading, from Eq. (8), to the acceleration of the constrained system

$$
\begin{aligned}
\ddot{\mathbf{x}}= & \left(\mathbf{M}^{-1}-\mathbf{M}^{-1} \mathbf{A}^{T}\left(\mathbf{A} \mathbf{M}^{-1} \mathbf{A}^{T}\right)^{-1} \mathbf{A} \mathbf{M}^{-1}\right) \mathbf{F}_{e} \\
& +\mathbf{M}^{-1} \mathbf{A}^{T}\left(\mathbf{A} \mathbf{M}^{-1} \mathbf{A}^{T}\right)^{-1} \mathbf{b} \\
= & \mathbf{M}^{-1} \mathbf{F}_{e}+\mathbf{M}^{-1} \mathbf{A}^{T}\left(\mathbf{A} \mathbf{M}^{-1} \mathbf{A}^{T}\right)^{-1}\left(\mathbf{b}-\mathbf{A} \mathbf{M}^{-1} \mathbf{F}_{e}\right)
\end{aligned}
$$

or

J. Acoust. Soc. Am. 141 (2), February 2017

$$
\mathbf{A} \ddot{\mathbf{x}}=\mathbf{b},
$$

where the $P \times M$ matrix $\mathbf{A}(\mathbf{x}(t), \dot{\mathbf{x}}(t), t)$ and $P \times 1$ vector $\mathbf{b}(\mathbf{x}(t), \dot{\mathbf{x}}(t), t)$ are functions of the motion. The $P$ constraints need not be independent, therefore, the rank $r$ of matrix $\mathbf{A}$ is $r \leq P$.

The dynamical solution $\mathbf{x}_{u}(t)$ of the unconstrained system is obviously given by

$$
\mathbf{M} \ddot{\mathbf{x}}_{u}=\mathbf{F}_{e} \Rightarrow \ddot{\mathbf{x}}_{u}=\mathbf{M}^{-1} \mathbf{F}_{e},
$$

while the response $\mathbf{x}(t)$ of the constrained system also depends on the vector $\mathbf{F}_{c}(t)$ of constraining forces

$$
\mathbf{M} \ddot{\mathbf{x}}=\mathbf{F}_{e}+\mathbf{F}_{c} .
$$

Using the method of Lagrange multipliers, see for instance Shabana (2010), the vector $\lambda(t)$ is defined such that

$$
\mathbf{F}_{c}=-\mathbf{A}^{T} \lambda
$$

or from Eq. (5),

$$
\mathbf{M} \ddot{\mathbf{x}}+\mathbf{A}^{T} \lambda=\mathbf{F}_{e}
$$

and, from Eqs. (3) and (7), the following augmented DAE of index one formulation may be built for the dynamics of the constrained system

$$
\left[\begin{array}{cc}
\mathbf{M} & \mathbf{A}^{T} \\
\mathbf{A} & \mathbf{0}
\end{array}\right]\left\{\begin{array}{l}
\ddot{\mathbf{x}} \\
\boldsymbol{\lambda}
\end{array}\right\}=\left\{\begin{array}{c}
\mathbf{F}_{e} \\
\mathbf{b}
\end{array}\right\}
$$

or, assuming the matrix is invertible (here, a nonsingular matrix implies nonzero masses in $\mathbf{M}$ and a full rank constraint matrix A), one formally obtains

$$
\left\{\begin{array}{l}
\ddot{\mathbf{x}} \\
\boldsymbol{\lambda}
\end{array}\right\}=\left[\begin{array}{cc}
\mathbf{M} & \mathbf{A}^{T} \\
\mathbf{A} & \mathbf{0}
\end{array}\right]^{-1}\left\{\begin{array}{c}
\mathbf{F}_{e} \\
\mathbf{b}
\end{array}\right\}
$$

Now, as noted by Arabyan and Wu (1998), from the matrix inversion identity
$\left.\begin{array}{c}\mathbf{M}^{-1} \mathbf{A}^{T}\left(\mathbf{A M}^{-1} \mathbf{A}^{T}\right)^{-1} \\ -\left(\mathbf{A} \mathbf{M}^{-1} \mathbf{A}^{T}\right)^{-1}\end{array}\right]$

$$
\ddot{\mathbf{x}}=\ddot{\mathbf{x}}_{u}+\mathbf{M}^{-1} \mathbf{A}^{T}\left(\mathbf{A} \mathbf{M}^{-1} \mathbf{A}^{T}\right)^{-1}\left(\mathbf{b}-\mathbf{A} \ddot{\mathbf{x}}_{u}\right),
$$

which shows the correction brought by the constraints to the unconstrained acceleration vector. On the other hand, one also obtains from Eq. (8) the Lagrange multipliers $\lambda(t)$,

$$
\begin{aligned}
\boldsymbol{\lambda} & =\left(\mathbf{A} \mathbf{M}^{-1} \mathbf{A}^{T}\right)^{-1} \mathbf{A} \mathbf{M}^{-1} \mathbf{F}_{e}-\left(\mathbf{A} \mathbf{M}^{-1} \mathbf{A}^{T}\right)^{-1} \mathbf{b} \\
& =-\left(\mathbf{A} \mathbf{M}^{-1} \mathbf{A}^{T}\right)^{-1}\left(\mathbf{b}-\mathbf{A} \mathbf{M}^{-1} \mathbf{F}_{e}\right),
\end{aligned}
$$


and, from Eqs. (6) and (13), the corresponding forces $\mathbf{F}_{c}(t)$ stemming from the constraints read

$$
\mathbf{F}_{c}=\mathbf{A}^{T}\left(\mathbf{A} \mathbf{M}^{-1} \mathbf{A}^{T}\right)^{-1}\left(\mathbf{b}-\mathbf{A} \mathbf{M}^{-1} \mathbf{F}_{e}\right) .
$$

Results (12) and (14) may already be traced to papers by Hemami and Weimer (1981) and Lötstedt (1982).

Defining now $\mathbf{B}(t)=\mathbf{A}(t) \mathbf{M}^{-1 / 2}$ one develops the coefficient of the second term in Eq. (12) as

$$
\begin{aligned}
\mathbf{M}^{-1} & \mathbf{A}^{T}\left(\mathbf{A} \mathbf{M}^{-1} \mathbf{A}^{T}\right)^{-1} \\
& =\mathbf{M}^{-1 / 2} \mathbf{M}^{-1 / 2} \mathbf{A}^{T}\left(\mathbf{A} \mathbf{M}^{-1 / 2} \mathbf{M}^{-1 / 2} \mathbf{A}^{T}\right)^{-1} \\
& =\mathbf{M}^{-1 / 2} \mathbf{B}^{T}\left(\mathbf{B} \mathbf{B}^{T}\right)^{-1}=\mathbf{M}^{-1 / 2} \mathbf{B}^{+}
\end{aligned}
$$

and the main result emerges

$$
\ddot{\mathbf{x}}=\ddot{\mathbf{x}}_{u}+\mathbf{M}^{-1 / 2} \mathbf{B}^{+}\left(\mathbf{b}-\mathbf{A} \ddot{\mathbf{x}}_{u}\right)
$$

where $\mathbf{B}^{+}$stands for the Moore-Penrose pseudo-inverse of matrix B, see, for instance, Golub and Van Loan (1996). On the other hand, from Eq. (14), the constraint forces are formulated as

$$
\mathbf{F}_{c}=\mathbf{M}^{1 / 2} \mathbf{B}^{+}\left(\mathbf{b}-\mathbf{A} \ddot{\mathbf{x}}_{u}\right) .
$$

The same result might be obtained multiplying Eq. (16) by the system mass matrix and accounting for Eq. (5),

$$
\begin{aligned}
\mathbf{M} \ddot{\mathbf{x}} & =\mathbf{M} \ddot{\mathbf{x}}_{u}+\mathbf{M} \mathbf{M}^{-1 / 2} \mathbf{B}^{+}\left(\mathbf{b}-\mathbf{A} \ddot{\mathbf{x}}_{u}\right) \\
& =\mathbf{M} \ddot{\mathbf{x}}_{u}+\mathbf{M}^{1 / 2} \mathbf{B}^{+}\left(\mathbf{b}-\mathbf{A} \ddot{\mathbf{x}}_{u}\right)=\mathbf{F}_{e}+\mathbf{F}_{c}
\end{aligned}
$$

Equations (16) and (17) are the basic results obtained by Udwadia and Kalaba, which may be applied to linear or nonlinear, conservative or dissipative systems. For a given excitation $\mathbf{F}_{e}(t)$, Eq. (16) may be efficiently solved using a suitable time-step integration scheme. The connection between Eq. (16) and other approaches, such as the GibbsAppell formulation, is provided by Udwadia (1996).

It may be seen that, if no constraints are applied, then the correcting term in Eq. (11) is nil and the unconstrained formulation (4) is recovered. The superlative elegance of the $\mathrm{U}-\mathrm{K}$ formulation (16) lies in the fact that it encapsulates, in a single explicit equation, both the dynamical equations of the system and the constraints applied. No additional variables, such as Lagrange multipliers, are needed. Furthermore, as pointed out by Arabyan and Wu (1998), due to the specific features of the Moore-Penrose pseudo-inverse, Eq. (16) always leads to constrained formulations in terms of standard ODE systems, even when non-independent or redundant constraints are used, and the inverse (9) does not exist, thus, avoiding numerically challenging mixed DAE systems.

The basic formulation (16) has been extended in various directions in order to deal with more general and challenging cases, namely, the alternative formulation developed by Udwadia and Phohomsiri (2006) to address systems that display singular mass matrices, the extension developed by
Udwadia and Kalaba (2000) to deal with work-performing non-ideal constraints, as well as the augmented formulations proposed by Yoon et al. (1994), Blajer (2002), and Braun and Goldfarb (2009) for enforcing lower-derivative constraints, thus, eliminating the possible residual drift of computed responses based on the higher-order acceleration constraint formulation.

\section{THE MODAL U-K FORMULATION}

We will now adapt the U-K formulation in order to deal with continuous flexible systems whose dynamics will be described in terms of modal coordinates. To convert formulation (16) in physical coordinates to the modal space, we start from the usual transformation

$$
\mathbf{x}=\Phi \boldsymbol{q}, \quad \dot{\mathbf{x}}=\Phi \dot{\boldsymbol{q}}, \quad \ddot{\mathbf{x}}=\Phi \ddot{\boldsymbol{q}}
$$

where, for $s=1,2, \ldots, S$ constrained subsystems, we define the vectors that assemble the corresponding physical responses $\mathbf{x}^{s}(t)$ and modal responses $\boldsymbol{q}^{s}(t)$, as well as the matrix that assembles the modeshapes $\Phi^{s}$,

$$
\mathbf{x} \equiv\left\{\begin{array}{c}
\mathbf{x}^{1} \\
\mathbf{x}^{2} \\
\vdots \\
\mathbf{x}^{S}
\end{array}\right\}, \quad \boldsymbol{q} \equiv\left\{\begin{array}{c}
\boldsymbol{q}^{1} \\
\boldsymbol{q}^{2} \\
\vdots \\
\boldsymbol{q}^{S}
\end{array}\right\}, \Phi \equiv\left[\begin{array}{cccc}
\Phi^{1} & \mathbf{0} & \cdots & \mathbf{0} \\
\mathbf{0} & \Phi^{2} & \cdots & \mathbf{0} \\
\vdots & \vdots & \ddots & \vdots \\
\mathbf{0} & \mathbf{0} & \cdots & \Phi^{S}
\end{array}\right]
$$

with, for each subsystem the modal basis consists of $N_{s}$ unconstrained modes

$$
\begin{gathered}
\boldsymbol{q}^{s}(t) \equiv\left\{\begin{array}{c}
q_{1}^{s}(t) \\
q_{2}^{s}(t) \\
\vdots \\
q_{N_{s}}^{s}(t)
\end{array}\right\}, \\
\left.\Phi^{s} \equiv\left[\begin{array}{c}
\phi_{1}^{s}\left(\vec{r}_{1}^{s}\right) \\
\phi_{1}^{s}\left(\vec{r}_{2}^{s}\right) \\
\vdots \\
\phi_{1}^{s}\left(\vec{r}_{R_{s}}^{s}\right)
\end{array}\right\}\left\{\begin{array}{c}
\phi_{2}^{s}\left(\vec{r}_{1}^{s}\right) \\
\phi_{2}^{s}\left(\vec{r}_{2}^{s}\right) \\
\vdots \\
\phi_{2}^{s}\left(\vec{r}_{R_{s}}^{s}\right)
\end{array}\right\} \cdots\left\{\begin{array}{c}
\phi_{N_{s}}^{s}\left(\vec{r}_{1}^{s}\right) \\
\phi_{N_{s}}^{s}\left(\vec{r}_{2}^{s}\right) \\
\vdots \\
\phi_{N_{s}}^{s}\left(\vec{r}_{R_{s}}^{s}\right)
\end{array}\right\}\right], \\
s=1,2, \ldots, S,
\end{gathered}
$$

the modeshapes being defined at $R$ physical coordinates. We then replace Eq. (19) into Eq. (12), so that

$$
\mathbf{M} \Phi \ddot{\boldsymbol{q}}=\mathbf{M} \Phi \ddot{\boldsymbol{q}}_{u}+\mathbf{A}^{T}\left(\mathbf{A} \mathbf{M}^{-1} \mathbf{A}^{T}\right)^{-1}\left(\mathbf{b}-\mathbf{A} \Phi \ddot{\boldsymbol{q}}_{u}\right) .
$$

Then, pre-multiplying Eq. (22) by the transpose of the modal matrix

$$
\Phi^{T} \mathbf{M} \Phi \ddot{\boldsymbol{q}}=\Phi^{T} \mathbf{M} \Phi \ddot{\boldsymbol{q}}_{u}+\Phi^{T} \mathbf{A}^{T}\left(\mathbf{A} \mathbf{M}^{-1} \mathbf{A}^{T}\right)^{-1}\left(\mathbf{b}-\mathbf{A} \Phi \ddot{\boldsymbol{q}}_{u}\right),
$$

and defining the matrix of modal masses $\boldsymbol{M}=\Phi^{T} \mathbf{M} \Phi$, hence, the corresponding inverse of the physical mass matrix $\mathbf{M}^{-1}=\Phi M^{-1} \Phi^{T}$ 


$$
\boldsymbol{M} \ddot{\boldsymbol{q}}=\boldsymbol{M} \ddot{\boldsymbol{q}}_{u}+\Phi^{T} \mathbf{A}^{T}\left(\mathbf{A} \Phi \boldsymbol{M}^{-1} \Phi^{T} \mathbf{A}^{T}\right)^{-1}\left(\mathbf{b}-\mathbf{A} \Phi \ddot{\boldsymbol{q}}_{u}\right),
$$

and introducing the modal constraint matrix $\boldsymbol{A}=\mathbf{A} \Phi$, we obtain

$$
\ddot{\boldsymbol{q}}=\ddot{\boldsymbol{q}}_{u}+\boldsymbol{M}^{-1} \boldsymbol{A}^{T}\left(\boldsymbol{A} \boldsymbol{M}^{-1} \boldsymbol{A}^{T}\right)^{-1}\left(\mathbf{b}-\boldsymbol{A}^{T} \ddot{\boldsymbol{q}}_{u}\right),
$$

we finally obtain, after defining $\boldsymbol{B}(t)=\boldsymbol{A}(t) \boldsymbol{M}^{-1 / 2}$,

$$
\ddot{\boldsymbol{q}}=\ddot{\boldsymbol{q}}_{u}+\boldsymbol{M}^{-1 / 2} \boldsymbol{B}^{+}\left(\mathbf{b}-\boldsymbol{A} \ddot{\boldsymbol{q}}_{u}\right) .
$$

This formulation in terms of the modal quantities is quite similar to the U-K formulation (16) in physical coordinates, except for the introduced changes leading to the modified constraint matrices $\boldsymbol{A}(t)$ and $\boldsymbol{B}(t)$.

Then, let us assume a set of $S$ vibrating subsystems, each one defined in terms of its unconstrained modal basis, which are coupled through $P$ kinematic constraints. The physical motions of the subsystems are governed by the usual modal equations

$$
\boldsymbol{M}^{s} \ddot{\boldsymbol{q}}^{s}+\boldsymbol{C}^{s} \dot{\boldsymbol{q}}^{s}+\boldsymbol{K}^{s} \boldsymbol{q}^{s}+\boldsymbol{F}_{n l}^{s}\left(\boldsymbol{q}^{s}, \dot{\boldsymbol{q}}^{s}\right)=\boldsymbol{F}_{\mathrm{ext}}^{s}, \quad s=1,2, \ldots, S .
$$

Here, for each subsystem $s$, the modal parameters in the diagonal matrices $\boldsymbol{M}^{s}, \boldsymbol{C}^{s}$, and $\boldsymbol{K}^{s}$ are given, respectively, as

$$
\left\{\begin{array}{l}
m_{n}^{s}=\int_{D_{s}} \rho\left(\vec{r}^{s}\right)\left[\phi_{n}^{s}\left(\vec{r}^{s}\right)\right]^{2} d \vec{r}^{s} \\
c_{n}^{s}=2 m_{n}^{s} \omega_{n}^{s} \zeta_{n}^{s} \\
k_{n}^{s}=m_{n}^{s}\left(\omega_{n}^{s}\right)^{2}
\end{array} \quad s=1,2, \ldots, S, n=1,2, \ldots, N_{s}\right.
$$

where $\rho\left(\vec{r}^{s}\right)$ is the mass density, $\omega_{n}^{s}$ are the modal circular frequencies, $\zeta_{n}^{s}$ are the modal damping ratios, and $\phi_{n}^{s}\left(\vec{r}^{s}\right)$ are the modeshapes of each subsystem. The modal forces $\boldsymbol{F}_{\mathrm{ext}}^{s}(t)$ are computed by projecting the external force field on the modeshapes

$$
\begin{aligned}
& F_{n}^{s}(t)=\int_{D_{s}} F_{\mathrm{ext}}\left(\vec{r}^{s}, t\right) \phi_{n}^{s}\left(\vec{r}^{s}\right) d \vec{r}^{s}, \quad s=1,2, \ldots, S, \\
& \quad n=1,2, \ldots, N_{s},
\end{aligned}
$$

in vector-matrix form

$$
\boldsymbol{F}_{\mathrm{ext}}^{s}=\left(\Phi_{\mathrm{ext}}^{s}\right)^{T} \mathbf{F}_{\mathrm{ext}}^{s}, \quad s=1,2, \ldots, S,
$$

where the columns of each matrix $\Phi_{\mathrm{ext}}^{s}$ are built from the modeshapes of the corresponding subsystem $s$ at the external excitation locations. On the other hand, for each subsystem, physical displacements $X^{s}\left(\vec{r}^{s}, t\right)$, velocities $\dot{X}^{s}\left(\vec{r}^{s}, t\right)$, and accelerations $\ddot{X}^{s}\left(\vec{r}^{s}, t\right)$ are obtained from modal superposition

$$
\begin{aligned}
& X^{s}\left(\vec{r}^{s}, t\right)=\sum_{n=1}^{N_{s}} \phi_{n}^{s}\left(\vec{r}^{s}\right) q_{n}^{s}(t), \dot{X}^{s}\left(\vec{r}^{s}, t\right)=\sum_{n=1}^{N_{s}} \phi_{n}^{s}\left(\vec{r}^{s}\right) \dot{q}_{n}^{s}(t), \\
& \ddot{X}^{s}\left(\vec{r}^{s}, t\right)=\sum_{n=1}^{N_{s}} \phi_{n}^{s}\left(\vec{r}^{s}\right) \ddot{q}_{n}^{s}(t), \quad s=1,2, \ldots, S,
\end{aligned}
$$

in vector-matrix form

$$
\mathbf{x}^{s}=\Phi^{s} \boldsymbol{q}^{s}, \quad \dot{\mathbf{x}}^{s}=\Phi^{s} \dot{\boldsymbol{q}}^{s}, \quad \ddot{\mathbf{x}}^{s}=\Phi^{s} \ddot{\boldsymbol{q}}^{s}, \quad s=1,2, \ldots, S .
$$

From Eq. (27) we obtain for each subsystem the unconstrained acceleration

$$
\ddot{\boldsymbol{q}}_{u}^{s}=\left(\boldsymbol{M}^{s}\right)^{-1} \boldsymbol{F}^{s}, \quad s=1,2, \ldots, S,
$$

where vector $\boldsymbol{F}^{s}(t)$ contains the contraint-independent modal forces, which include the term $\boldsymbol{F}_{\text {ext }}^{s}(t)$ stemming from the external motion-independent force field, Eq. (29), as well as those related to the linear and nonlinear modal dissipative and elastic forces

$$
\boldsymbol{F}^{s}=\boldsymbol{F}_{\mathrm{ext}}^{s}-\boldsymbol{C}^{s} \dot{\boldsymbol{q}}^{s}-\boldsymbol{K}^{s} \boldsymbol{q}^{s}-\boldsymbol{F}_{n l}^{s}\left(\boldsymbol{q}^{s}, \dot{\boldsymbol{q}}^{s}\right), \quad s=1,2, \ldots, S,
$$

where the vectors of modal constrained displacements and velocities are assumed known at each time-step.

With respect to formulation (26), with Eqs. (33) and (34), we further define the following assembled vectors and matrices of modal quantities:

$$
\begin{aligned}
& \ddot{\boldsymbol{q}} \equiv\left\{\begin{array}{c}
\ddot{\boldsymbol{q}}^{1} \\
\ddot{\boldsymbol{q}}^{2} \\
\vdots \\
\ddot{\boldsymbol{q}}^{S}
\end{array}\right\} ; \quad \ddot{\boldsymbol{q}}_{u} \equiv\left\{\begin{array}{c}
\ddot{\boldsymbol{q}}_{u}^{1} \\
\ddot{\boldsymbol{q}}_{u}^{2} \\
\vdots \\
\ddot{\boldsymbol{q}}_{u}^{S}
\end{array}\right\} ; \boldsymbol{M} \equiv\left[\begin{array}{cccc}
\boldsymbol{M}^{1} & \mathbf{0} & \cdots & \mathbf{0} \\
\mathbf{0} & \boldsymbol{M}^{2} & \cdots & \mathbf{0} \\
\vdots & \vdots & \ddots & \vdots \\
\mathbf{0} & \mathbf{0} & \cdots & \boldsymbol{M}^{S}
\end{array}\right], \\
& \boldsymbol{C} \equiv\left[\begin{array}{cccc}
\boldsymbol{C}^{1} & \mathbf{0} & \cdots & \mathbf{0} \\
\mathbf{0} & \boldsymbol{C}^{2} & \cdots & \mathbf{0} \\
\vdots & \vdots & \ddots & \vdots \\
\mathbf{0} & \mathbf{0} & \cdots & \boldsymbol{C}^{S}
\end{array}\right] ; \boldsymbol{K} \equiv\left[\begin{array}{cccc}
\boldsymbol{K}^{1} & \mathbf{0} & \cdots & \mathbf{0} \\
\mathbf{0} & \boldsymbol{K}^{2} & \cdots & \mathbf{0} \\
\vdots & \vdots & \ddots & \vdots \\
\mathbf{0} & \mathbf{0} & \cdots & \boldsymbol{K}^{S}
\end{array}\right],
\end{aligned}
$$

and the unconstrained modal accelerations $\ddot{\boldsymbol{q}}_{u}(t)$ are computed as

$$
\begin{aligned}
\left\{\begin{array}{c}
\ddot{\boldsymbol{q}}_{u}^{1} \\
\ddot{\boldsymbol{q}}_{u}^{2} \\
\vdots \\
\ddot{\boldsymbol{q}}_{u}^{S}
\end{array}\right\}= & {\left[\begin{array}{cccc}
\left(\boldsymbol{M}^{1}\right)^{-1} & \mathbf{0} & \cdots & \mathbf{0} \\
\mathbf{0} & \left(\boldsymbol{M}^{2}\right)^{-1} & \cdots & \mathbf{0} \\
\vdots & \vdots & \ddots & \vdots \\
\mathbf{0} & \mathbf{0} & \cdots & \left(\boldsymbol{M}^{S}\right)^{-1}
\end{array}\right] } \\
& +\left[\begin{array}{c}
\boldsymbol{F}_{\mathrm{ext}}^{1} \\
\boldsymbol{F}_{\mathrm{ext}}^{2} \\
\vdots \\
\boldsymbol{F}_{\mathrm{ext}}^{S}
\end{array}\right\}-\left[\begin{array}{cccc}
\boldsymbol{C}^{1} & \mathbf{0} & \cdots & \mathbf{0} \\
\mathbf{0} & \boldsymbol{C}^{2} & \cdots & \mathbf{0} \\
\vdots & \vdots & \ddots & \vdots \\
\mathbf{0} & \mathbf{0} & \cdots & \boldsymbol{C}^{S}
\end{array}\right]\left\{\begin{array}{c}
\dot{\boldsymbol{q}}^{1} \\
\dot{\boldsymbol{q}}^{2} \\
\vdots \\
\dot{\boldsymbol{q}}^{S}
\end{array}\right\} \\
& {\left.\left[\begin{array}{cccc}
\boldsymbol{K}^{1} & \mathbf{0} & \cdots & \mathbf{0} \\
\mathbf{0} & \boldsymbol{K}^{2} & \cdots & \mathbf{0} \\
\vdots & \vdots & \ddots & \vdots \\
\mathbf{0} & \mathbf{0} & \cdots & \boldsymbol{K}^{S}
\end{array}\right]\left\{\begin{array}{c}
\boldsymbol{q}^{1} \\
\boldsymbol{q}^{2} \\
\vdots \\
\boldsymbol{q}^{S}
\end{array}\right\}-\left\{\begin{array}{c}
\boldsymbol{F}_{n l}^{1}\left(\boldsymbol{q}^{1}, \dot{\boldsymbol{q}}^{1}\right) \\
\boldsymbol{F}_{n l}^{2}\left(\boldsymbol{q}^{2}, \dot{\boldsymbol{q}}^{2}\right) \\
\vdots \\
\boldsymbol{F}_{n l}^{S}\left(\boldsymbol{q}^{S}, \dot{\boldsymbol{q}}^{S}\right)
\end{array}\right\}\right) . }
\end{aligned}
$$


Turning now to the $P$ constraints, these are amenable to linear (often time-changing) relationships of the type

$$
\boldsymbol{A} \ddot{\boldsymbol{q}}=\mathbf{b},
$$

with $\boldsymbol{A}(\boldsymbol{q}(t), \dot{\boldsymbol{q}}(t), t)$ and $\boldsymbol{b}(\boldsymbol{q}(t), \dot{\boldsymbol{q}}(t), t)$ defined at specific constraint locations $\vec{r}_{c}^{s}$ between the subsystems. These will be written as

$$
\begin{gathered}
{\left[\begin{array}{c}
A_{1}\left(\Phi^{1}\left(\vec{r}_{1}^{1}\right), \ldots, \Phi^{S}\left(\vec{r}_{1}^{S}\right) ; \boldsymbol{q}^{1}, \ldots, \boldsymbol{q}^{S} ; \dot{\boldsymbol{q}}^{1}, \ldots, \dot{\boldsymbol{q}}^{S} ; t\right) \\
A_{2}\left(\Phi^{1}\left(\vec{r}_{2}^{1}\right), \ldots, \Phi^{S}\left(\vec{r}_{2}^{S}\right) ; \boldsymbol{q}^{1}, \ldots, \boldsymbol{q}^{S} ; \dot{\boldsymbol{q}}^{1}, \ldots, \dot{\boldsymbol{q}}^{S} ; t\right) \\
\vdots \\
A_{P}\left(\Phi^{1}\left(\vec{r}_{P}^{1}\right), \ldots, \Phi^{S}\left(\vec{r}_{P}^{S}\right) ; \boldsymbol{q}^{1}, \ldots, \boldsymbol{q}^{S} ; \dot{\boldsymbol{q}}^{1}, \ldots, \dot{\boldsymbol{q}}^{S} ; t\right)
\end{array}\right]} \\
\times\left\{\begin{array}{c}
\ddot{\boldsymbol{q}}^{1} \\
\ddot{\boldsymbol{q}}^{2} \\
\vdots \\
\ddot{\boldsymbol{q}}^{S}
\end{array}\right\}=\left\{\begin{array}{c}
b_{1}\left(\boldsymbol{q}^{1}, \ldots, \boldsymbol{q}^{S} ; \dot{\boldsymbol{q}}^{1}, \ldots, \dot{\boldsymbol{q}}^{S} ; t\right) \\
b_{2}\left(\boldsymbol{q}^{1}, \ldots, \boldsymbol{q}^{S} ; \dot{\boldsymbol{q}}^{1}, \ldots, \dot{\boldsymbol{q}}^{S} ; t\right) \\
\vdots \\
b_{P}\left(\boldsymbol{q}^{1}, \ldots, \boldsymbol{q}^{S} ; \dot{\boldsymbol{q}}^{1}, \ldots, \dot{\boldsymbol{q}}^{S} ; t\right)
\end{array}\right\} .
\end{gathered}
$$

The transient response of the constrained system can be obtained through the following strategy:

(1) Computation (or experimental identification) of the modal parameters $m_{n}^{s}, \omega_{n}^{s}, \zeta_{n}^{s}$, and $\phi_{n}^{s}\left(\vec{r}^{s}\right)$ for each unconstrained subsystem;

(2) At any time-step, an explicit numerical solution of the constrained system is obtained

(a) First by computing the modal forces independent from the constraints $\boldsymbol{F}^{s}\left(\boldsymbol{q}^{s}, \dot{\boldsymbol{q}}^{s}, t\right)$, Eq. (34);

(b) Then by computing the modal accelerations $\ddot{\boldsymbol{q}}_{u}(t)$ of the unconstrained system, Eq. (33);

(c) Then by computing the modal accelerations $\ddot{\boldsymbol{q}}(t)$ of the constrained system, Eq. (26);

(d) Finally by performing the propagation of the modal solutions to the next time-step using some suitable integration algorithm.

(3) Physical responses at any location $\vec{r}^{s}$ may be obtained by superposition of the modal responses, Eqs. (31) or (32).

Finally, if needed, the physical constraining forces $\mathbf{F}_{c}(t)$ may be also computed as follows. From Eq. (26) the modal forces $\boldsymbol{F}_{c}(t)$ due to the constraints are computed by multiplying the modal acceleration complement $\ddot{\boldsymbol{q}}_{c}=\boldsymbol{M}^{-1 / 2} \boldsymbol{B}^{+}$ $\left(\mathbf{b}-\boldsymbol{A} \ddot{\boldsymbol{q}}_{u}\right)$ by the system modal mass matrix $\boldsymbol{M}$, hence,

$$
\boldsymbol{F}_{c}=\boldsymbol{M}^{1 / 2} \boldsymbol{B}^{+}\left(\mathbf{b}-\boldsymbol{A} \ddot{\boldsymbol{q}}_{u}\right),
$$

and conversion of the modal constraint forces to the physical constraining forces is achieved through the following approximation:

$$
\mathbf{F}_{c}=\left(\left(\Phi_{c}\right)^{T}\right)^{+} \boldsymbol{F}_{c}
$$

hence,

$$
\mathbf{F}_{c}=\left(\left(\Phi_{c}\right)^{T}\right)^{+} \boldsymbol{M}^{1 / 2} \boldsymbol{B}^{+}\left(\mathbf{b}-\boldsymbol{A} \ddot{\boldsymbol{q}}_{u}\right) .
$$

\section{GUITAR STRING/BODY/PLAYER COUPLING}

As an illustration, we now address the coupled dynamics of a guitar string and body, coupled at the instrument bridge, as well as stopped by a finger for note-tuning purposes or a capodastro, somewhere on the fingerboard. The vibrating continuous string and instrument body will be modelled using the U-K formulation combined with a modal discretization of the instrument components, using the unconstrained modal basis of the string and instrument body. A single string will be addressed in the present demonstrative computations, nevertheless extension to a full set of coupled strings is achievable as shown by the authors; see Marques et al. (2013) and Debut et al. (2014, 2015, 2016). Significant work has been produced by several authors on guitar modeling, using modal methods, as well as finite-element and finite-difference computational approaches; see Woodhouse (2004a,b) and Derveaux et al. (2003) for particularly representative contributions, the former work offering extensive modeling and experiments, the latter also dealing with sound radiation.

The basic system studied is the guitar illustrated in Fig. 1, which highlights the strings termination at the thin bridge glued to the guitar soundboard and shows the relevant quantities used for modeling. From then on, quantities pertaining to the string will be referred to as subscript or superscript $S$, while quantities pertaining to the instrument body will be denoted $B$. Let us consider the response $Y^{S}(x, t)$ of a plucked guitar string with total length $L$ (from nut to bridge), subjected to constraints, respectively: (a) at the bridge location $x_{B}=L$, where it is coupled to the instrument soundboard, and (b) at one or several locations $x_{F}$ of a stopping finger on the fingerboard. The string will be assumed excited at location $x_{E}$ by imposing a near-point time-varying force such as might be obtained from a plectrum.

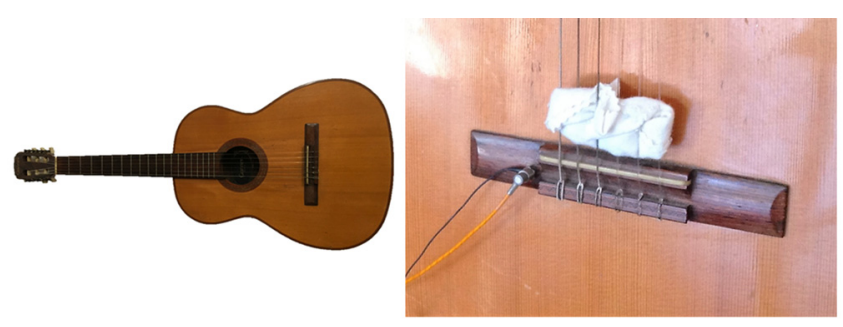

(a)

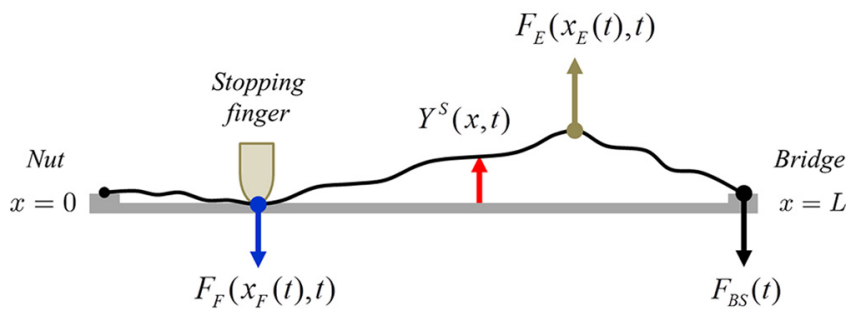

(b)

FIG. 1. (Color online) (a) Giannini tested guitar (upper left); string/body coupling through the guitar bridge, picture also showing the transducers used for measuring the body transfer function at the bridge (upper right); (b) description of the relevant quantities for physical modeling. 


\section{A. String/body coupling at the bridge}

The bridge is comparatively hard and massive, as compared to the flexibility of both the string and soundboard, and will therefore be treated here as a rigid transmission interface. For small vibration amplitudes, the string and body geometric and material nonlinearities may well be assumed negligible, therefore, the basic unconstrained modal Eq. (36) reads

$$
\begin{aligned}
\left\{\begin{array}{c}
\ddot{\boldsymbol{q}}_{u}^{S} \\
\ddot{\boldsymbol{q}}_{u}^{B}
\end{array}\right\}= & {\left[\begin{array}{cc}
\left(\boldsymbol{M}^{S}\right)^{-1} & \mathbf{0} \\
\mathbf{0} & \left(\boldsymbol{M}^{B}\right)^{-1}
\end{array}\right] } \\
& \times\left(-\left[\begin{array}{cc}
\boldsymbol{C}^{S} & \mathbf{0} \\
\mathbf{0} & \boldsymbol{C}^{B}
\end{array}\right]\left\{\begin{array}{c}
\dot{\boldsymbol{q}}^{S} \\
\dot{\boldsymbol{q}}^{B}
\end{array}\right\}\right. \\
& \left.-\left[\begin{array}{cc}
\boldsymbol{K}^{S} & \mathbf{0} \\
\mathbf{0} & \boldsymbol{K}^{B}
\end{array}\right]\left\{\begin{array}{c}
\boldsymbol{q}^{S} \\
\boldsymbol{q}^{B}
\end{array}\right\}+\left\{\begin{array}{c}
\boldsymbol{F}_{\mathrm{exc}}^{S} \\
\mathbf{0}
\end{array}\right\}\right),
\end{aligned}
$$

where the external modal forces $\boldsymbol{F}_{\text {exc }}^{S}(t)$ stem from the string playing, at location(s) $x_{E}(t)$, where the excitation force $F_{E}\left(x_{E}(t), t\right)$ is applied. No external body forces $\boldsymbol{F}_{\text {exc }}^{B}(t)$ are considered, as the string/body coupling is formulated in terms of a constraint equation: The bridge location $x_{B}=L$, the string motion $Y^{S}\left(x_{B}, t\right)$ must be the same as the instrument body motion at the string location $Y^{B}\left(\vec{r}_{S}, t\right)$. Then, two different cases will be studied in the following:

(a1) The body is rigid and motionless (as in a monochord):

$$
Y^{S}\left(x_{B}, t\right)=0 \Rightarrow\left(\Phi_{B}^{S}\right)^{T} \boldsymbol{q}^{S}(t)=0 .
$$

(a2) The body is flexible and defined in terms of its modal basis

$$
\begin{aligned}
& Y^{S}\left(x_{B}, t\right)-Y^{B}\left(\vec{r}_{S}, t\right)=0 \\
& \quad \Rightarrow\left(\Phi_{B}^{S}\right)^{T} \boldsymbol{q}^{S}(t)-\left(\Phi_{S}^{B}\right)^{T} \boldsymbol{q}^{B}(t)=0 .
\end{aligned}
$$

Obviously, in constraints (43) and (44) the modeshape vector is taken at the relevant locations $\Phi_{B}^{S} \equiv \Phi^{S}\left(x_{B}\right)$ and $\Phi_{S}^{B} \equiv \Phi^{B}\left(\vec{r}_{S}\right)$. The modal excitation vector in Eq. (42) is obtained by modal projection of $F_{E}\left(x_{E}(t), t\right)$ on the string modes, as per Eqs. (29) and (30). For generality, we assume that the excitation location(s) $x_{E}(t)$ may change in time, following a musician's playing.

\section{B. String/finger coupling at the soundboard}

For simplicity, the string/finger coupling model is thought of as a rigid kinematical constraint, at a single location (see Fig. 2). Such an assumed constraint directly enters into the realm of the standard kinematical constraints common in multibody theory. At the location $x_{F}(t)$ modeling a stopping finger on the fingerboard, the string motion $Y^{S}\left(x_{F}(t), t\right)$ should be nil, $\forall t$,

$$
Y^{S}\left(x_{F}(t), t\right)=0 \Rightarrow\left(\Phi_{F}^{S}(t)\right)^{T} \boldsymbol{q}^{S}(t)=0,
$$

where $\Phi_{F}^{S}(t) \equiv \Phi^{S}\left(x_{F}(t)\right)$ stands for the string single stopping locations. Notice that for generality we assume that the finger location(s) $x_{F}(t)$, may change in time, following the musician's sound-tuning during playing.

\section{Dynamical formulation}

For the hypothesis of rigid constraining of the string at the fingerboard, from Eqs. (43) and (45) the condensed constraint formulation (38) becomes

$$
\boldsymbol{A}(t)\left\{\begin{array}{c}
\ddot{\boldsymbol{q}}^{S}(t) \\
\ddot{\boldsymbol{q}}^{B}(t)
\end{array}\right\}=\mathbf{b}(t),
$$

with

$$
\begin{aligned}
& \boldsymbol{A}_{(a 1)}(t) \equiv\left[\begin{array}{cc}
\left(\Phi_{B}^{S}\right)^{T} & \mathbf{0}^{T} \\
\left(\Phi_{F}^{S}(t)\right)^{T} & \mathbf{0}^{T}
\end{array}\right] ; \\
& \boldsymbol{A}_{(a 2)}(t) \equiv\left[\begin{array}{cc}
\left(\Phi_{B}^{S}\right)^{T} & -\left(\Phi_{S}^{B}\right)^{T} \\
\left(\Phi_{F}^{S}(t)\right)^{T} & \mathbf{0}^{T}
\end{array}\right] ; \quad \mathbf{b}(t) \equiv\left\{\begin{array}{c}
0 \\
0 \\
\vdots \\
0
\end{array}\right\} .
\end{aligned}
$$

Finally, for this problem (and other similar instruments), the constrained modal accelerations are readily computed as

$$
\left\{\begin{array}{c}
\ddot{\boldsymbol{q}}^{S} \\
\ddot{\boldsymbol{q}}^{B}
\end{array}\right\}=\boldsymbol{W}(t)\left\{\begin{array}{c}
\ddot{\boldsymbol{q}}_{u}^{S} \\
\ddot{\boldsymbol{q}}_{u}^{B}
\end{array}\right\},
$$

with the global constraint-enforcing matrix

$$
\boldsymbol{W}(t)=\mathbf{1}-\boldsymbol{M}^{-1 / 2} \boldsymbol{B}(t)^{+} \boldsymbol{A}(t) .
$$

If needed, the string and instrument body responses may be recovered at any time and location from Eqs. (31) and (32). Concerning the modal constraining forces $\boldsymbol{F}_{c}(t)$, these are obtained from Eq. (39) as

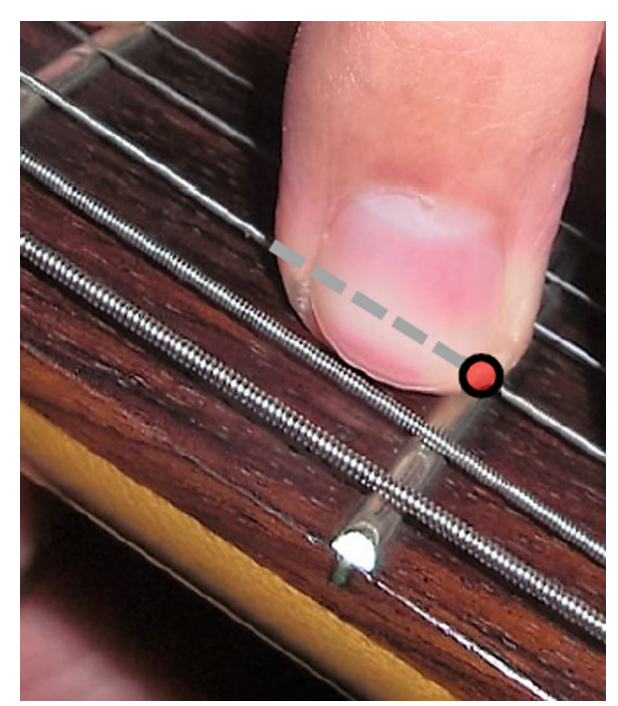

FIG. 2. (Color online) String/finger coupling model: Rigid kinematical constraint at the fret location. 


$$
\left\{\begin{array}{c}
\boldsymbol{F}_{c}^{S} \\
\boldsymbol{F}_{c}^{B}
\end{array}\right\}=\boldsymbol{Z}(t)\left\{\begin{array}{c}
\ddot{\boldsymbol{q}}_{u}^{S} \\
\ddot{\boldsymbol{q}}_{u}^{B}
\end{array}\right\},
$$

with

$$
\boldsymbol{Z}(t)=-\boldsymbol{M}^{1 / 2} \boldsymbol{B}(t)^{+} \boldsymbol{A}(t),
$$

and, from Eq. (50), the physical constraining forces $F_{c}(t)$ are obtained through Eq. (40).

Notice that, if the matrix of modal constraints $\boldsymbol{A}$ is timeindependent (e.g., the stopping finger does not move), then matrices $\boldsymbol{W}$ and $\boldsymbol{Z}$ are also constant and may be computed once and for all, so that the constraint-enforcing equation (48) is numerically quite efficient. Even if several different notes are played in succession, the limited number of matrices $\boldsymbol{W}_{\text {note }}$ can be pre-computed prior to the time-loop, so that obtaining the dynamical responses remains quite efficient. Nevertheless, care should be taken when implementing sequences of notes, in order not to enforce rigid constraints to the string at the successive finger positions without insuring a smooth transition of the string dynamics at each new note. Such issue will be addressed elsewhere.

Obviously, any number of strings may be easily coupled to the body by extending the relevant dynamical and constraint operators as appropriate, thus, modeling a complete instrument. Then, to compute the time-domain responses, many adequate ODE solvers may be used. Here, a simple explicit velocity-Verlet algorithm was implemented; see, for instance, Press et al. (2007), and details of the implementation are given in the Appendix.

\section{ILLUSTRATIVE COMPUTATIONS}

\section{A. System parameters}

The illustrative computations presented in the following are based on guitar body modes experimentally identified from a transfer function we measured at the instrument bridge; see Fig. 1. The computed string $\left(A_{2}\right)$ is tuned to a fundamental of $f_{1}=110 \mathrm{~Hz}$, with length (from nut to bridge) $L=0.65 \mathrm{~m}$, axial tensioning force $T=73.9 \mathrm{~N}$, mass per unit length $\rho_{l}=3.61 \times 10^{-3} \mathrm{~kg} / \mathrm{m}$, transverse wave propagation velocity $c_{t}=\sqrt{T / \rho_{l}}=143 \mathrm{~m} / \mathrm{s}$, and inharmonicity parameter (bending stiffness of a non-ideal string) $B=E I=4$ $\times 10^{-5} \mathrm{Nm}^{2}$. These parameters have been taken from the experimental work by Woodhouse (2004b). Then, for the unconstrained string used in our dynamical computations (pinned at the nut and "free" at the bridge), the inharmonic modal frequencies are computed as

$$
f_{n}^{S}=\frac{c_{t}}{2 \pi} p_{n}\left(1+\frac{B}{2 T} p_{n}^{2}\right) \text { with } p_{n}=\frac{(2 n-1) \pi}{2 L}
$$

with modeshapes:

$$
\phi_{n}^{S}(x)=\sin \left(\frac{(2 n-1) \pi x}{2 L}\right),
$$

and modal masses

$$
m_{n}^{S}=\rho_{l} \int_{0}^{L}\left[\phi_{n}^{S}(x)\right]^{2} d x=\frac{\rho_{l} L}{2}, \quad \forall n
$$

Concerning the string modal damping, complex dissipative phenomena must be accounted for, as thoroughly discussed by Woodhouse (2004a,b), who proposed the following pragmatic formulation for modal damping based on three loss parameters:

$$
\zeta_{n}^{S}=\frac{1}{2} \frac{T\left(\eta_{F}+\frac{\eta_{A}}{2 \pi f_{n}^{S}}\right)+\eta_{B} B p_{n}^{2}}{T+B p_{n}^{2}}
$$

where the loss coefficients, somewhat loosely described as "internal friction," "air viscous damping," and "bending damping," fitted from experimental data, are for this string $\eta_{F}=7 \times 10^{-5}, \eta_{A}=0.9$, and $\eta_{B}=2.5 \times 10^{-2}$.

Figure 3 illustrates the modal parameters of the unconstrained string for some modes. The black dot in the plots indicates the bridge location, where all unconstrained modes display an antinode. As shown in Eq. (54) the modal masses $m_{n}^{s}=0.012 \mathrm{~kg}$, based on modeshapes normalized as per expression (53), are identical for all string modes. Notice that, as should be expected, none of the unconstrained string modes of this modal basis approaches the fundamental frequency $110 \mathrm{~Hz}$ of the pinned-pinned string. However, when these modes are coupled by the string/body constraint at the bridge, the fundamental frequency will be recovered, as it should. Figure 4 collapses the modal frequencies and damping values for the first 100 modes of the string unconstrained sat the bridge. One can notice the rise of string modal damping at lower frequencies.

For the guitar body, modal parameters were identified in the frequency range $0-800 \mathrm{~Hz}$ from a transfer function (with both excitation and vibratory response normal to the soundboard) measured at the bridge of our tested instrument. Modal identification was achieved in the frequency domain by developing a multi-degree-of-freedom algorithm based upon a curve fitting procedure; see Johansson (1993). Starting with a set of initial modal parameters defined for a selection of $N$ resonances, the algorithm minimizes a global error between the measured transfer functions and the estimation model in the least-squares sense; see Allemang and Brown (1994). The modal parameters are shown in the Table I for the 16 modes identified used in the dynamical computations, as well as in Fig. 5. Notice that the modal masses given in Table I pertain to body modes normalized to unity amplitude at the bridge location.

\section{B. Response of the rigidly mounted string}

Starting with the basic kinematical constraint at the bridge, assumed for the moment motionless due to a rigid body, we computed the string response with a tuning string constraint on the fingerboard at $x=0.33 L$, using a single kinematic constraint $Y^{S}\left(x_{F}, t\right)=0$, for a simulated time of $10 \mathrm{~s}$ and using an integration time-step of $10^{-5} \mathrm{~s}$. The string 

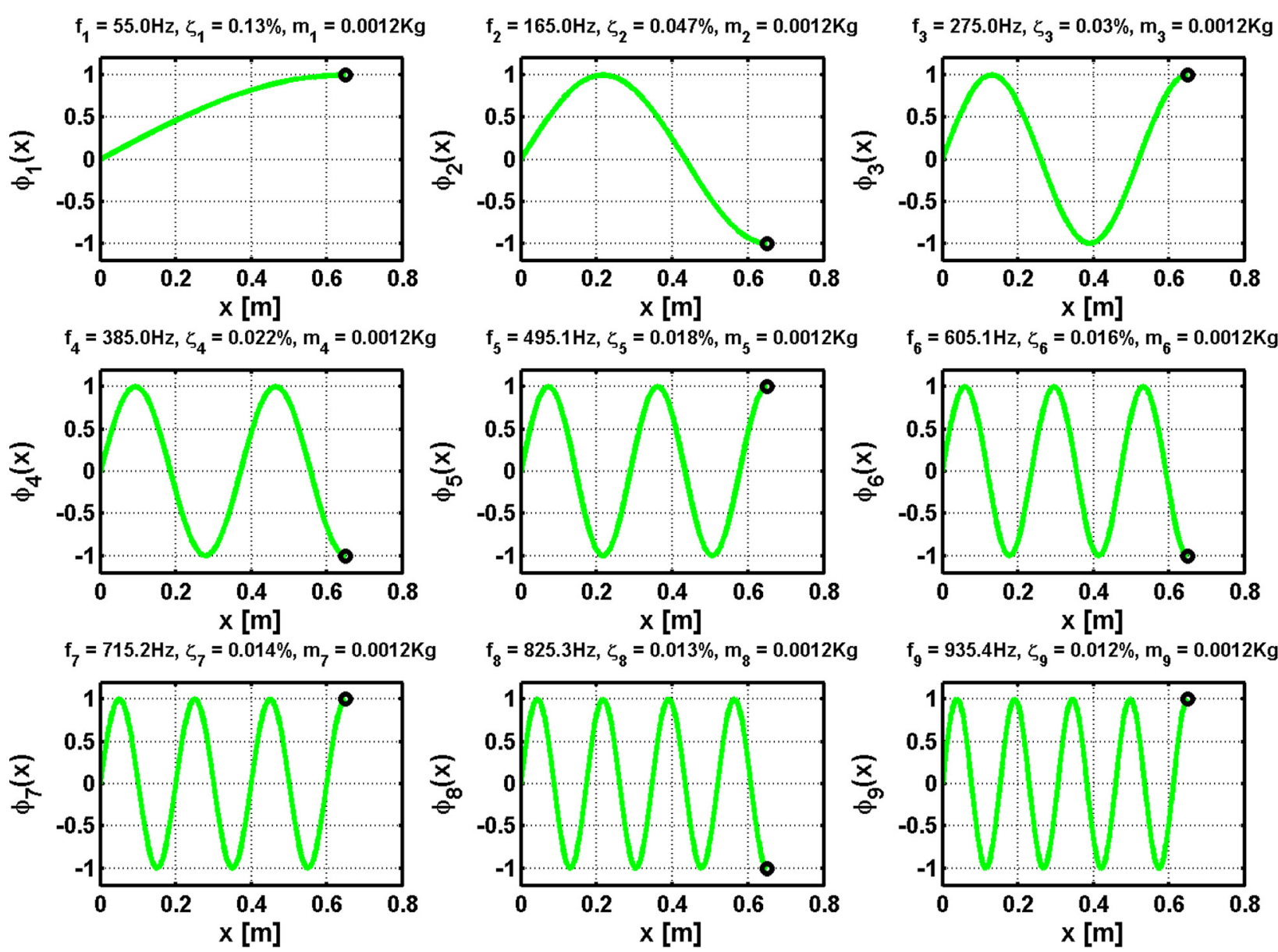

FIG. 3. (Color online) Sample modal parameters of the unconstrained string.

excitation is a linear force ramp $0-5 \mathrm{~N}$, applied near the bridge at $x_{E}=0.9 L$, for the initial $0.01 \mathrm{~s}$ of the simulation. For simplicity, a single motion polarization of the string is addressed here. Figure 6 shows the convergence of the computed string response, as the number of string modes is increased from 50 up to 200 , corresponding to maximum string modal frequencies ranging from about $5500 \mathrm{~Hz}$ up to $27500 \mathrm{~Hz}$. These results show that, indeed, the string motion
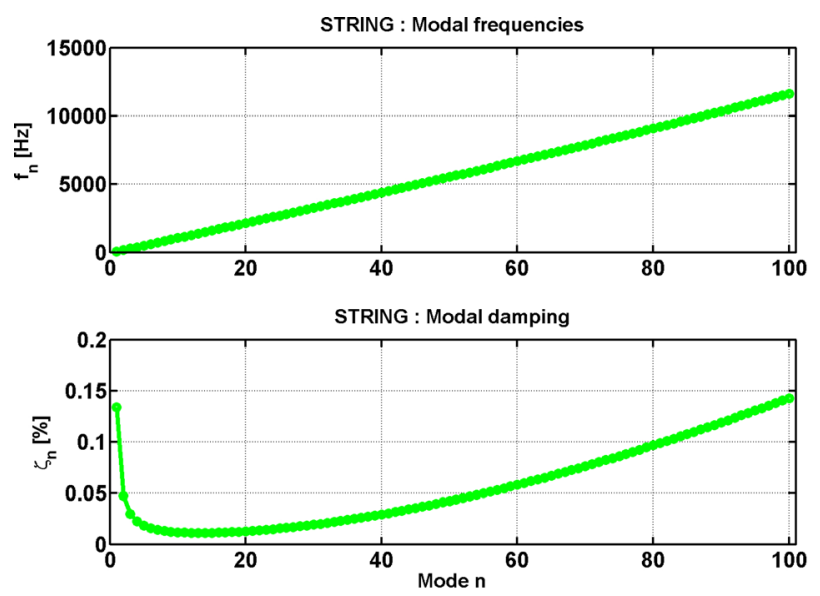

FIG. 4. (Color online) Modal frequencies and damping values of the unconstrained string. at both constraint locations (rigid bridge and finger) is virtually nil as expected. On the other hand, for this type of "hard" constraint, a relatively high number of modes must be used to achieve convergence of the simulated responses. In the following all simulations are performed using 150 string modes, up to about $20000 \mathrm{~Hz}$, which proved a sensible compromise.

TABLE I. Identified modal properties of the body.

\begin{tabular}{lccc}
\hline \hline Mode $n$ & $f_{n}(\mathrm{~Hz})$ & $\zeta_{n}(\%)$ & $m_{n}(\mathrm{~kg})$ \\
\hline 1 & 78.3 & 2.2 & 2.91 \\
2 & 100.2 & 1.1 & 0.45 \\
3 & 187.3 & 1.6 & 0.09 \\
4 & 207.8 & 1.0 & 0.25 \\
5 & 250.9 & 0.7 & 2.65 \\
6 & 291.8 & 0.9 & 9.88 \\
7 & 314.7 & 1.1 & 8.75 \\
8 & 344.5 & 0.7 & 8.80 \\
9 & 399.0 & 1.4 & 0.90 \\
10 & 429.6 & 0.9 & 0.41 \\
11 & 482.9 & 0.7 & 0.38 \\
12 & 504.2 & 0.7 & 1.07 \\
13 & 553.9 & 0.6 & 2.33 \\
14 & 580.3 & 1.4 & 1.36 \\
15 & 645.7 & 1.0 & 2.02 \\
16 & 723.5 & 1.3 & 0.45 \\
\hline \hline
\end{tabular}



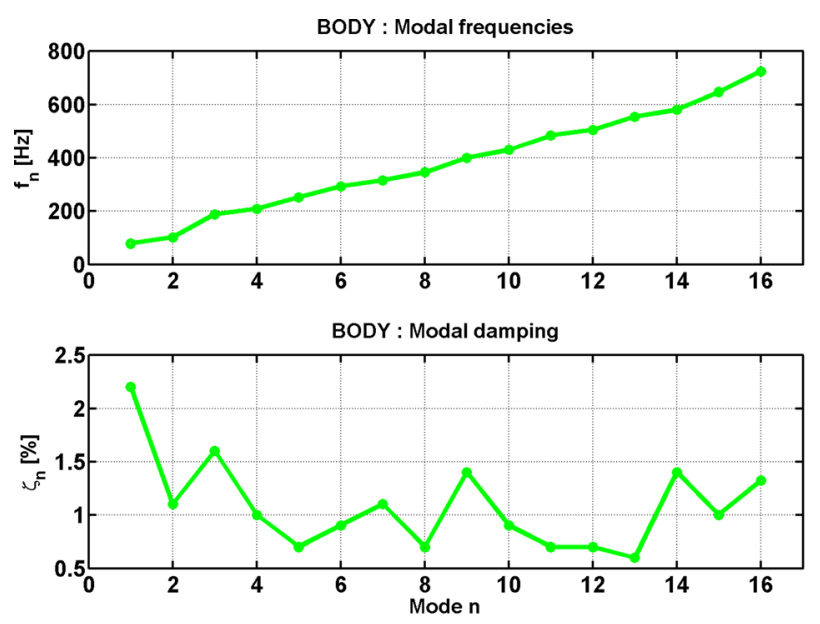

FIG. 5. (Color online) Modal frequencies and damping values of the instrument body.

Other aspects of the string response are highlighted in Fig. 7. The response spectrum shows typical nearly equally spaced response pics (as well as the residual spectra of the constrained rigid body and finger). Notice that, in spite of using the unconstrained pinned-free modal basis, when the
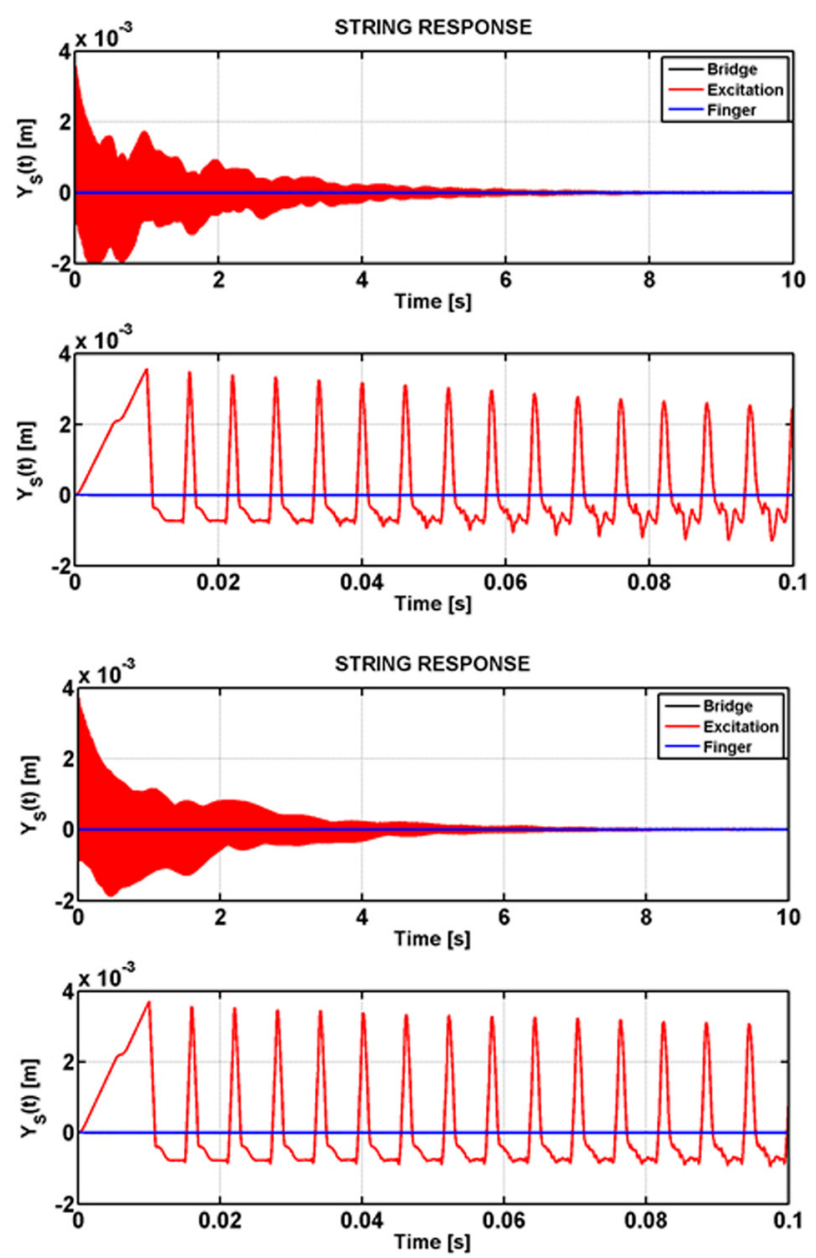

string is constrained at the rigid bridge, we recover the expected fundamental frequency for the pinned-pinned string $f_{1}^{A_{2}}=f_{1}^{S}\left(L-x_{E}\right) / L=165 \times 0.667=110 \mathrm{~Hz}$. Both coupling forces, at the bridge and stopping finger (or fret), are of the same order of magnitude of the maximum excitation force $(5 \mathrm{~N})$. They clearly display pulses that correspond to wave reflexions at the bridge and the finger, the pulse delay between both sides being half-period of the played fundamental, as expected. Finally, notice that, prior to each main reflexion pulse, higher frequency fluctuations arise, corresponding to the arrival of precursor flexural waves as the modelled string is not ideal.

Because in this computation the finger/fret is simulated through a single rigid constraint, energy can flow easily from the excited region of the string to the nominally "passive" region, and back. Such energy flow may be accentuated for both numerical or physical reasons. If the modal basis used in the computation is severely truncated, the string model becomes unduly rigidified, leading to unphysical energy transfer through the constraint. However, such energy transfer can be justified on physical ground as a result of the bending rigidity encapsulated in the string model. The waterfall time-space plot of Fig. 8 illustrates this effect by highlighting wave propagation phenomena along the string
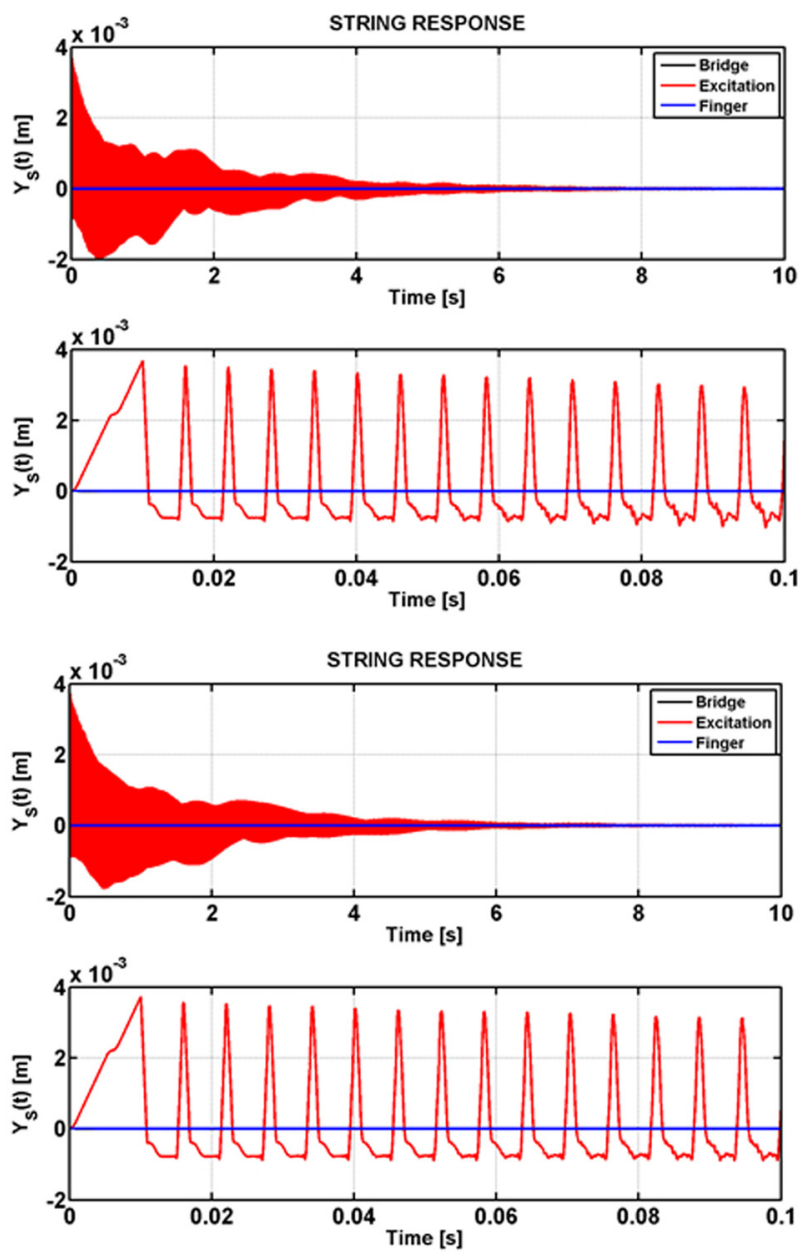

FIG. 6. (Color online) Computed string response for a string excited at $x_{E}=0.9 L$ and stopped at $x_{F}=0.33 L$ using a single rigid constraint (shown are the total simulated response and a detail of the starting transient): Computation using 50 string modes (upper left), 100 string modes (upper right), 150 string modes (lower left), and 200 string modes (lower right). 
for the first cycles of the computed motion, as well as by the end of the simulation. Wave reflection and transmission effects are most clear in the first plot, while their cumulative result already captured significant energy to the main string motion in the second plot.

The effects of this energy transfer may be felt by the instrument through the dynamical string force at the bridge. The spectrogram of the computed force shown in Fig. 9 clearly shows periodic fluctuations at partials 2, 4, 6, etc. These are clearly related to the modes 1,2 , 3 , etc. of the "passive" region, which have the same frequencies for the chosen stopping finger location $\left(x_{F}=0.33 L\right)$.

\section{Influence of the string/body coupling}

To conclude the present illustrative computations, we now introduce the dynamics of the instrument body, which are coupled to the string at the bridge, moving with the soundboard. The identified modal parameters of the body have been detailed in Sec. VA. The results obtained are shown in Figs. 10-12.
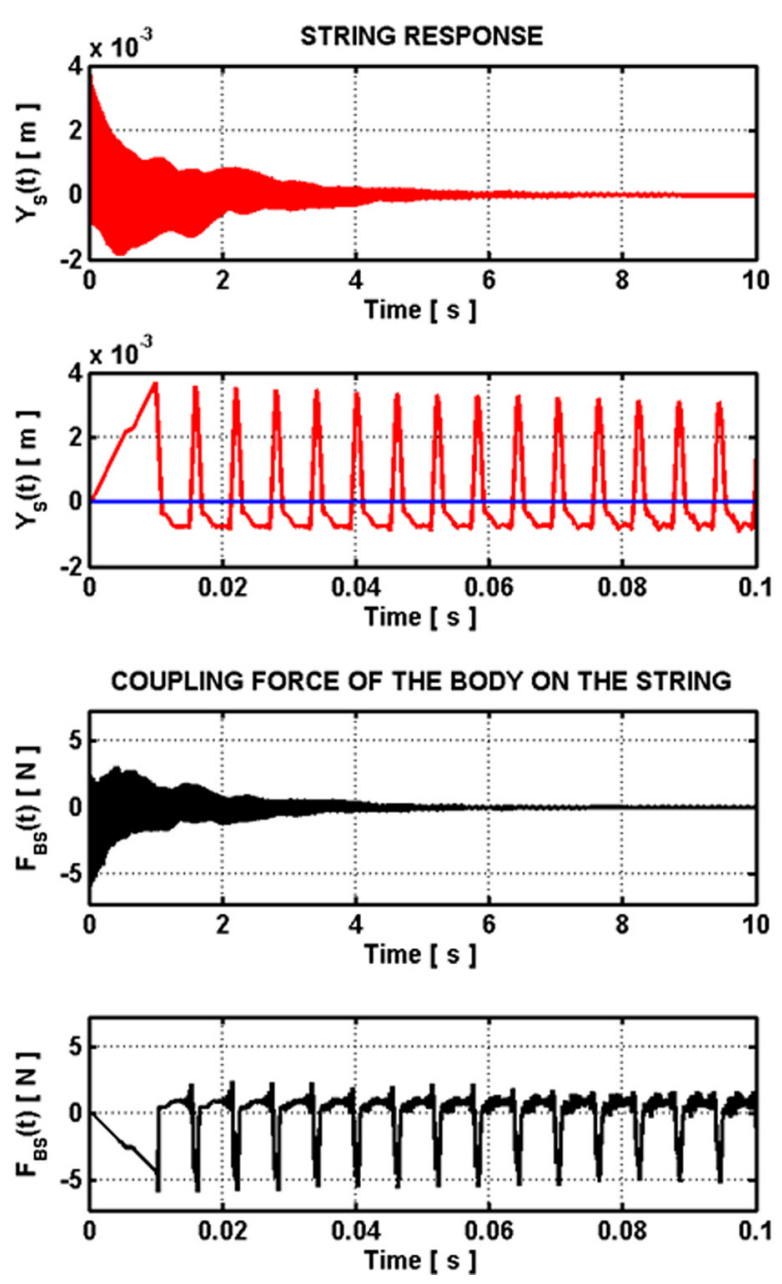

A comparison between Figs. 7 and 10 clearly highlights the influence of the body dynamics on the string response. In particular, the string decrease of motion amplitude in time, which is essentially exponential for the rigid support condition, becomes more complex when the body dynamics are included as energy is exchanged between them. Also, the global dissipative role of the instrument body clearly shows through the shorter life of the string response in Fig. 10. On the other hand, one can also notice peaks in the response spectra, which stem from the body modes.

The body dynamics barely affect the starting motion of the string, as can be asserted from the first time-space plots of Figs. 8 and 11. However, in the long run, the string motion is highly affected by the body modes, as documented by the later time-space plots of Figs. 8 and 11, which show totally different responses. Notice, in particular, the different rates of energy dissipation between the excited and the "passive" regions of the string, which reflect the string/body energy transfer. Finally, the influence of the body dynamics on the string responses is also supported when comparing the spectrograms of the reaction force at the bridge, Figs. 9 and 12, which clearly show different decays for the lower frequency partials.

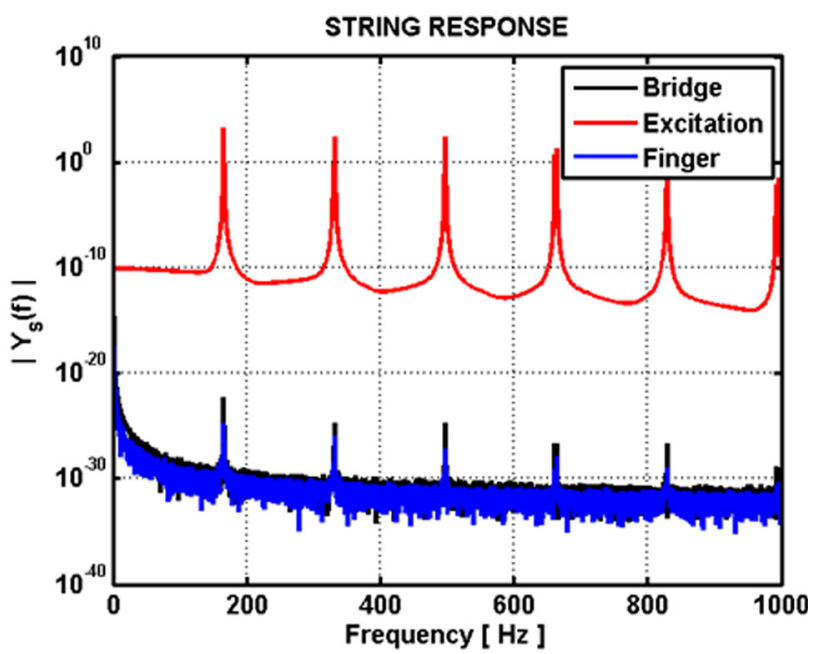

COUPLING FORCE OF THE FINGER ON THE STRING
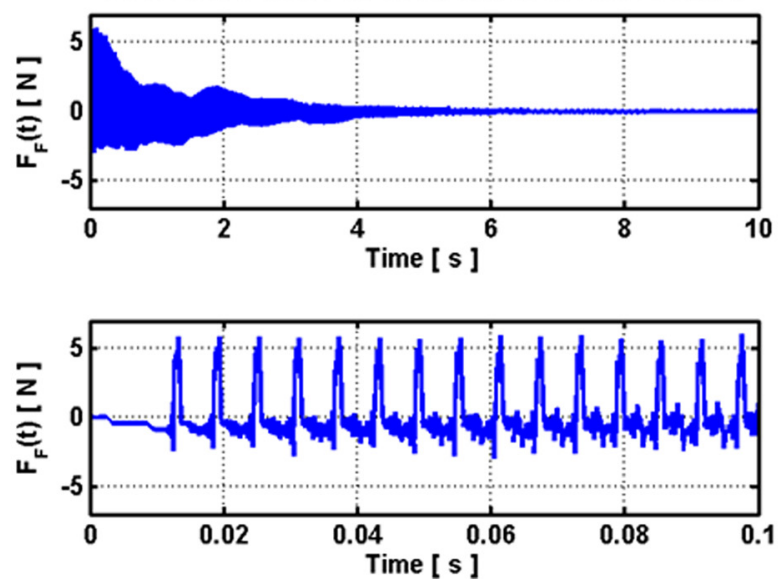

FIG. 7. (Color online) Several aspects of the computed string response for a string excited at $x_{E}=0.9 \mathrm{~L}$ and stopped at $x_{F}=0.33 \mathrm{~L}$ through a single rigid constraint (150 string modes; rigid body): Time-domain string response (upper left), corresponding response spectra (upper right), coupling force at the rigid bridge (lower left), and coupling force at the fingerboard (lower right). 


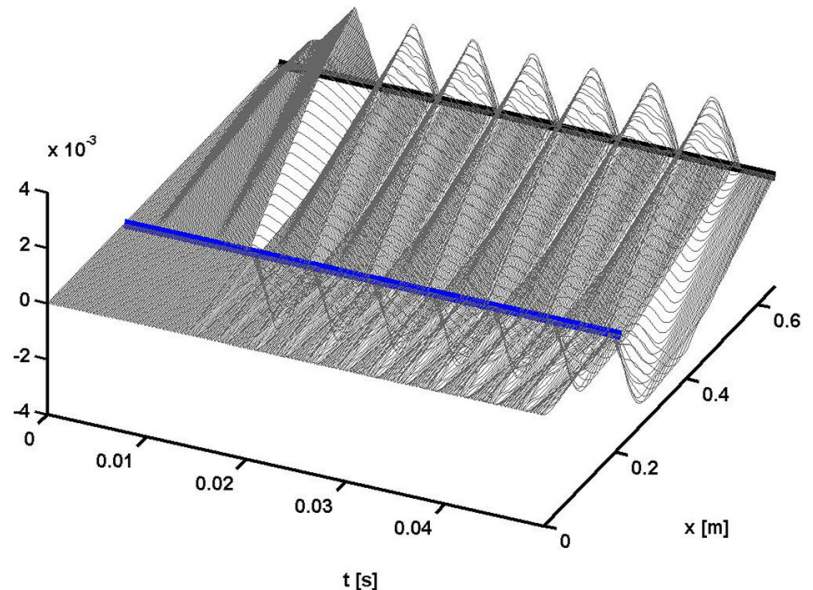

(a)

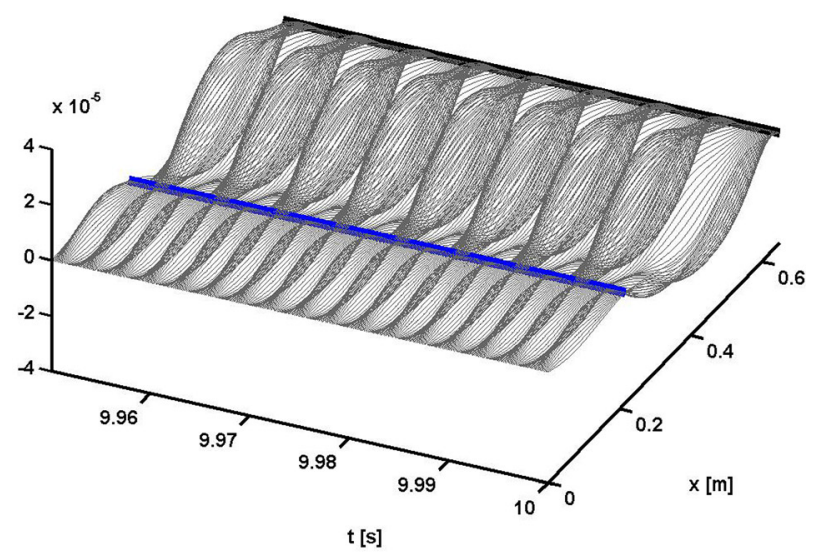

(b)

FIG. 8. (Color online) Time-space waterfall plot of the computed string response for a string excited at $x_{E}=0.9 \mathrm{~L}$ and stopped at $x_{F}=0.33 \mathrm{~L}$ through a single rigid constraint (150 string modes; rigid body): First cycles of the motion (up), motion at the end of the simulation (bottom).

\section{Comparison between the U-K and penalty-based formulations}

It is finally of interest to check the agreement of the responses computed by the U-K formulation with the corresponding results stemming from another modeling strategy. For the sake of comparison, we present a different approach to coupling, based on penalty formulation, following the lines presented by the authors for modeling string instruments; see Marques et al. (2013) and Debut et al. (2014, 2015, 2016). Instead of using kinematical constraints, penalty methods deal with coupling by introducing contact forces corresponding to the reactions of suitable springs and dampers, acting between interacting subsystems. This formulation is elegantly simple and easy to implement, but care must be taken for the choice of the penalty parameters to correctly enforce coupling and prevent numerical instabilities.

In view of penalty-based formulation, the dynamics of the fully coupled string/finger/body system is given in terms of modal coordinates by

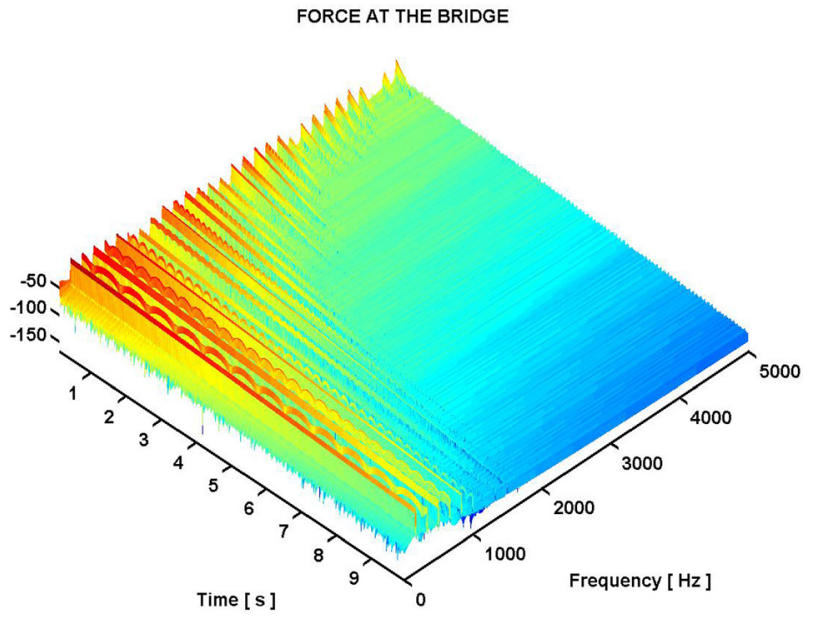

FIG. 9. (Color online) Spectrogram of the dynamical string reaction force at the bridge for a string excited at $x_{E}=0.9 \mathrm{~L}$ and stopped at $x_{F}=0.33 \mathrm{~L}$ through a single rigid constraint (150 string modes; rigid body).

$$
\begin{aligned}
& {\left[\begin{array}{cc}
\boldsymbol{M}^{S} & \mathbf{0} \\
\mathbf{0} & \boldsymbol{M}^{B}
\end{array}\right]\left\{\begin{array}{l}
\ddot{\boldsymbol{q}}^{S} \\
\ddot{\boldsymbol{q}}^{B}
\end{array}\right\}+\left[\begin{array}{cc}
\boldsymbol{C}^{S} & \mathbf{0} \\
\mathbf{0} & \boldsymbol{C}^{B}
\end{array}\right]\left\{\begin{array}{c}
\dot{\boldsymbol{q}}^{S} \\
\dot{\boldsymbol{q}}^{B}
\end{array}\right\}} \\
& +\left[\begin{array}{cc}
\boldsymbol{K}^{S} & \mathbf{0} \\
\mathbf{0} & \boldsymbol{K}^{B}
\end{array}\right]\left\{\begin{array}{l}
\boldsymbol{q}^{S} \\
\boldsymbol{q}^{B}
\end{array}\right\}=\left\{\begin{array}{l}
\boldsymbol{F}_{c}^{S} \\
\boldsymbol{F}_{c}^{B}
\end{array}\right\}+\left\{\begin{array}{c}
\boldsymbol{F}_{\mathrm{exc}}^{S} \\
\mathbf{0}
\end{array}\right\} .
\end{aligned}
$$

Again $\boldsymbol{M}^{S}, \boldsymbol{C}^{S}, \boldsymbol{K}^{S}$ and $\boldsymbol{M}^{B}, \boldsymbol{C}^{B}$, and $\boldsymbol{K}^{B}$ are the matrices of the modal parameters of the string and instrument body, respectively, and $\boldsymbol{q}^{S}(t)$ and $\boldsymbol{q}^{B}(t)$ are the corresponding modal response vectors. Besides the external modal forces $\boldsymbol{F}_{\text {exc }}^{S}$ corresponding to the string excitation, the right-hand side of Eq. (56) includes the modal forces $\boldsymbol{F}_{c}^{S}$ and $\boldsymbol{F}_{c}^{B}$, which relate to the local effects of the coupling interaction for the string and body, respectively. As usual, these modal interactions stem from the projections on the modal basis of the physical forces, here $F_{B S}(t)$ and $F_{F}(t)$, which are computed using the following penalty formulation as

$$
\begin{aligned}
F_{B S}(t)= & -K_{B S}\left(Y^{S}\left(x_{B}, t\right)-Y^{B}\left(\vec{r}_{S}, t\right)\right) \\
& -C_{B S}\left(\dot{Y}^{S}\left(x_{B}, t\right)-\dot{Y}^{B}\left(\vec{r}_{S}, t\right)\right), \\
F_{F}(t)= & -K_{F} Y^{S}\left(x_{F}(t), t\right)-C_{F} \dot{Y}^{S}\left(x_{F}(t), t\right),
\end{aligned}
$$

and where we introduce the penalty parameters $K_{B S}$ and $C_{B S}$ (respectively, $K_{F}$ and $C_{F}$ ) for the string/bridge (respectively, string/fret) interaction, and for which the fret is assumed motionless. Finally, the string/body and string/fret modal forces to be used in Eq. (56) are written as

$$
\begin{aligned}
\boldsymbol{F}_{c}^{S}(t) & =\Phi_{B}^{S} F_{B S}(t)+\Phi_{F}^{S}(t) F_{F}(t), \\
\boldsymbol{F}_{c}^{B}(t) & =\Phi_{S}^{B} F_{B S}(t) .
\end{aligned}
$$

For comparison between methods, the test case relates to the fully coupled model studied in Sec. V C, so that all the complexity of the dynamics is retained. Identical computational parameters were used in the two implementations, and numerical integrations were performed using the same 

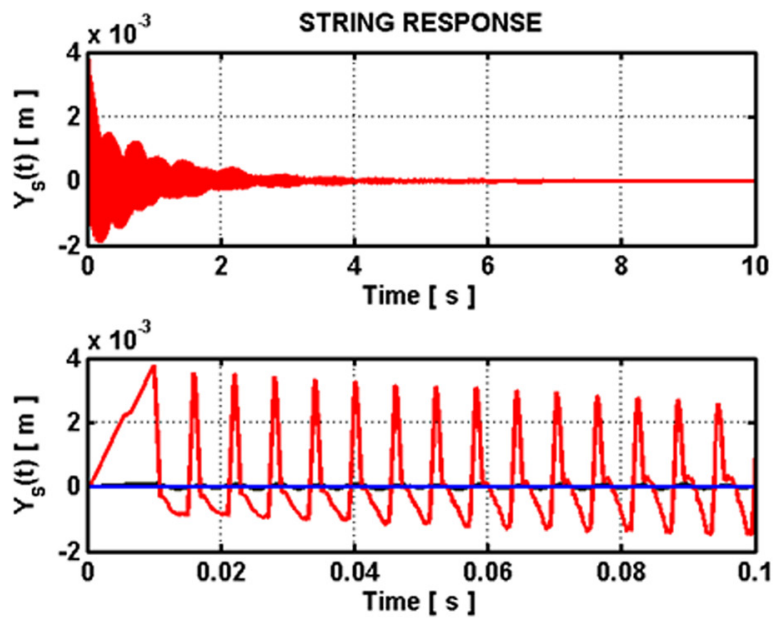

COUPLING FORCE OF THE BODY ON THE STRING
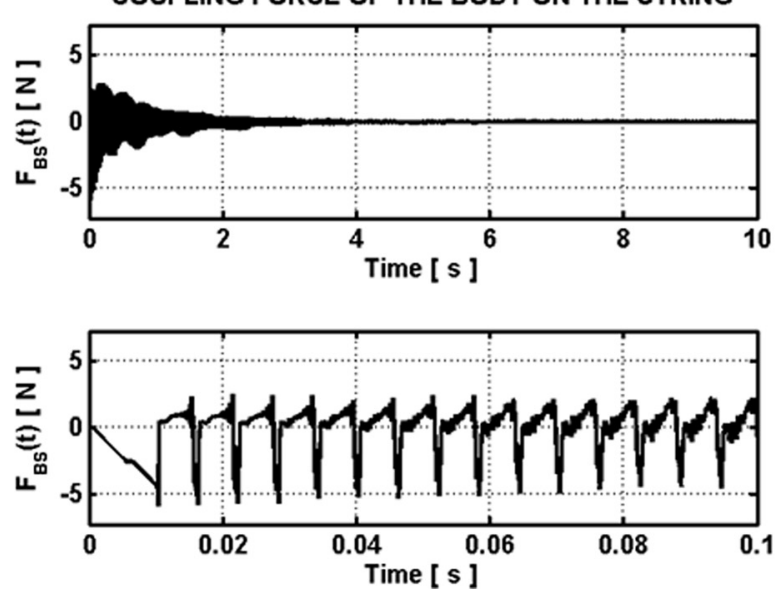
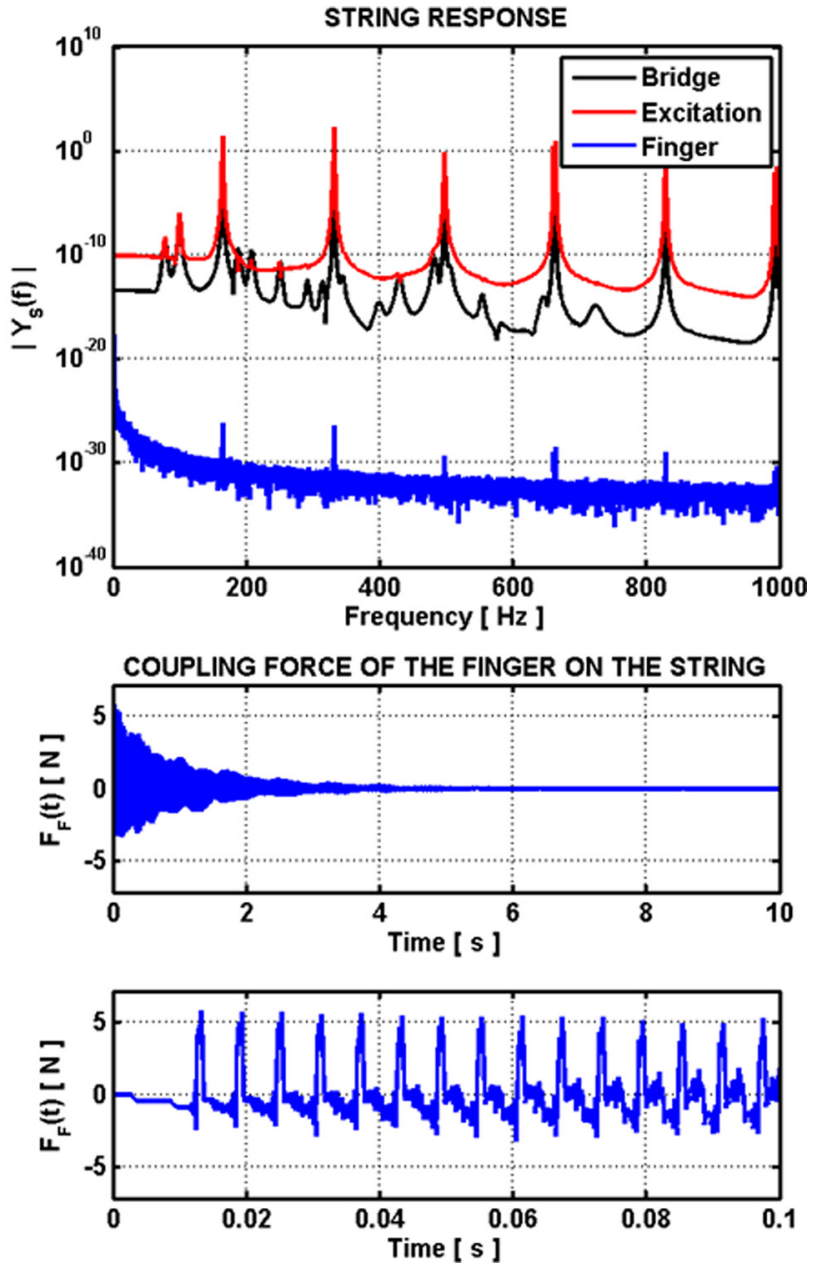

FIG. 10. (Color online) Several aspects of the computed string response for a string excited at $x_{E}=0.9 L$ and stopped at $x_{F}=0.33 L$ through a single rigid constraint (150 string modes; 16 body modes): Time-domain string response (upper left), corresponding response spectra (upper right), coupling force at the rigid bridge (lower left), and coupling force at the fingerboard (lower right).

integration scheme (velocity-Verlet). For the penalty formulation, values of $K_{B S}=K_{F}=10^{6} \mathrm{~N} / \mathrm{m}$ and $C_{B S}=C_{F}$ $=10^{-1} \mathrm{Ns} / \mathrm{m}$ were considered in order to simulate nearly rigid coupling at the connection points, and a convenient time-step of $10^{-7} \mathrm{~s}$ was used for ensuring stable behavior of the explicit numerical scheme.

Detailed simulation comparisons of the two implementations are presented in Figs. 13 and 14, where the string responses $Y^{S}(x, t)$ computed at several locations and the interaction forces $F_{B S}(t)$ and $F_{F}(t)$ are plotted. As seen, there is an excellent agreement between the responses stemming from the two methods. On the one hand, the string motion at the bridge location, as well as the interaction forces, are nearly identical. At the stopping finger, however, the string motions are noticeably different since the penalty-based formulation provides less stringent enforcement of the constraint than the kinematic condition. Nevertheless, the penalty approach enforces near-zero string displacement at the finger coupling point, which leads there to negligibly small-amplitude vibrations for the string. Besides the accurate agreement of predictions between the two methods, one important comment that can be made about the comparison concerns the computational time-steps. In the present computations, the penalty-based implementation requires a much smaller time-step for convergence for comparable results with a difference of 2 orders of magnitude in the time-step size. This is a real advantage of the U-K formulation compared to the penalty approach, in particular, for physical modeling sound synthesis of musical instruments.

\section{CONCLUSIONS}

In this paper, we developed a new approach for computing the dynamics of coupled flexible systems based on the general formulation of $\mathrm{U}-\mathrm{K}$, which is becoming increasingly popular in the field of multibody dynamics. The general U-K equation was adapted to address flexible coupled subsystems, linear or nonlinear, defined in terms of their unconstrained modal basis.

The U-K formulation shows a considerable potential to deal effectively with the dynamics of physically modelled musical instruments. Therefore, the formulation developed was applied to a guitar, including the fully coupled dynamics of a string, tuned by a stopping finger somewhere on the fingerboard, and the instrument body. The illustrative 


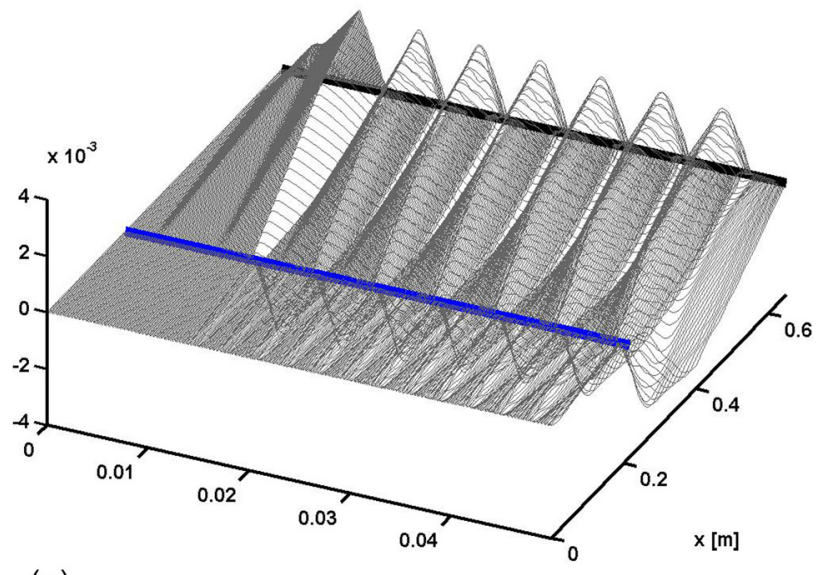

(a)

$t[s]$

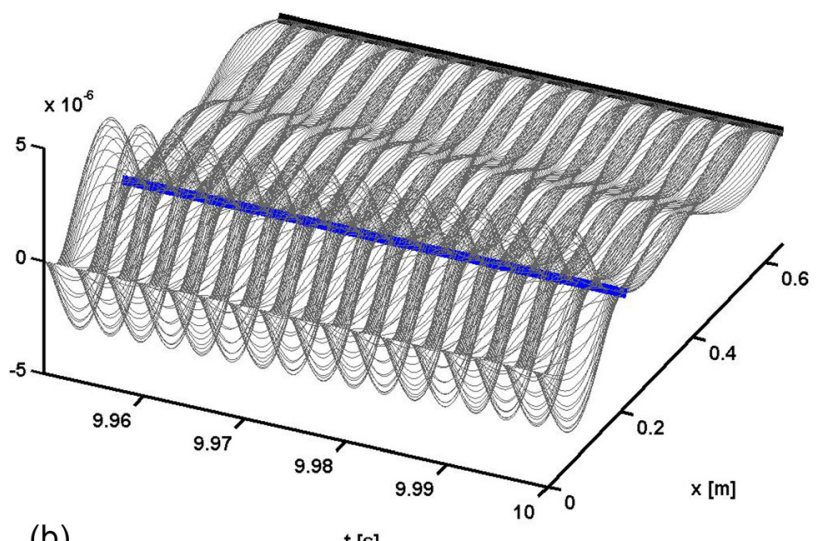

(b)

$\mathrm{t}[\mathrm{s}]$

FIG. 11. (Color online) Time-space waterfall plots of the computed string response for a string excited at $x_{E}=0.9 L$ and stopped at $x_{F}=0.33 L$ through one rigid constraint simulating the fret, followed by four flexible-dissipative-inertial constraints simulating the finger (150 string modes; 16 body modes): First cycles of the motion (up), motion at the end of the simulation (bottom).

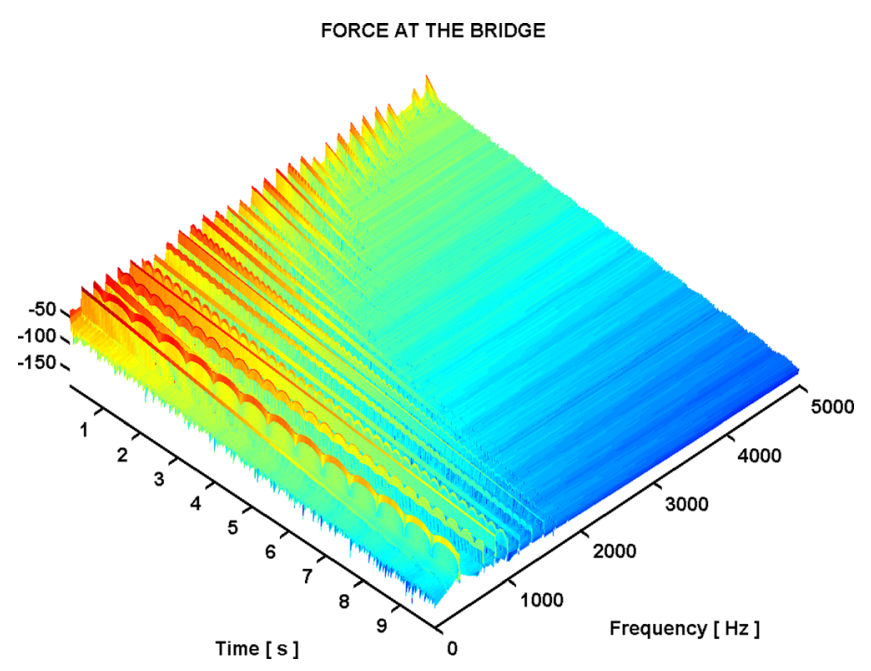

FIG. 12. (Color online) Spectrogram of the dynamical string reaction force at the bridge for a string excited at $x_{E}=0.9 \mathrm{~L}$ and stopped at $x_{F}=0.33 \mathrm{~L}$ through a single rigid constraint ( 150 string modes; 16 body modes).
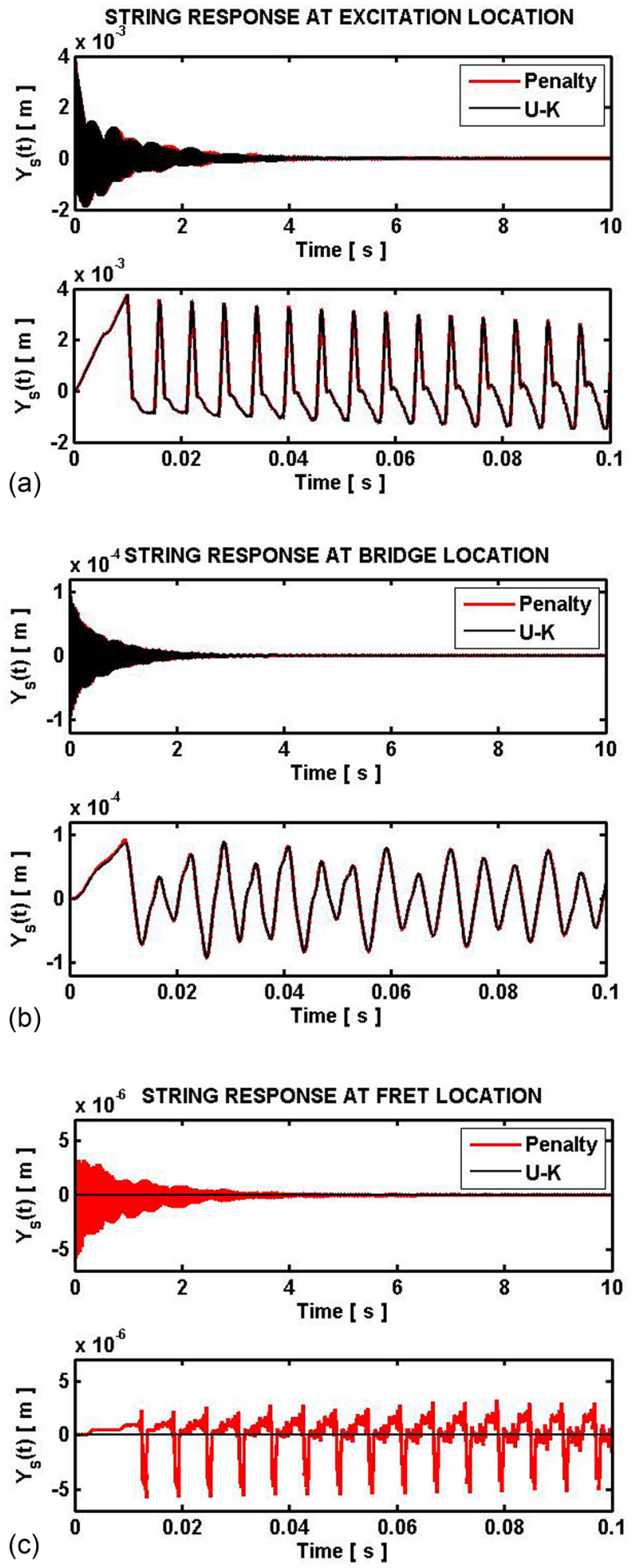

FIG. 13. (Color online) Comparison of the string responses at various locations, computed using the U-K implementation (black) and the penaltybased formulation (red), for a string excited at $x_{E}=0.9 \mathrm{~L}$ and stopped at $x_{F}=0.33 L$ (shown are the total simulated response and a detail of the starting transient): at the excitation location (top), at the bridge (middle), and the fret location (bottom). 150 string modes; 16 body modes.

computations presented highlight the role of the body dynamics on the string response, which significantly affects the vibratory responses of both the "active" and "passive" regions of the string. 
COUPLING FORCE OF THE BODY ON THE STRING
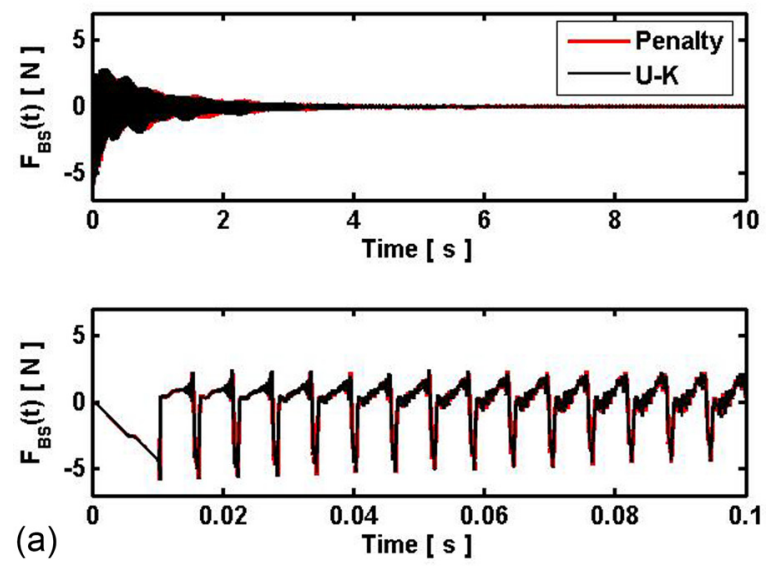

COUPLING FORCE OF THE FINGER ON THE STRING
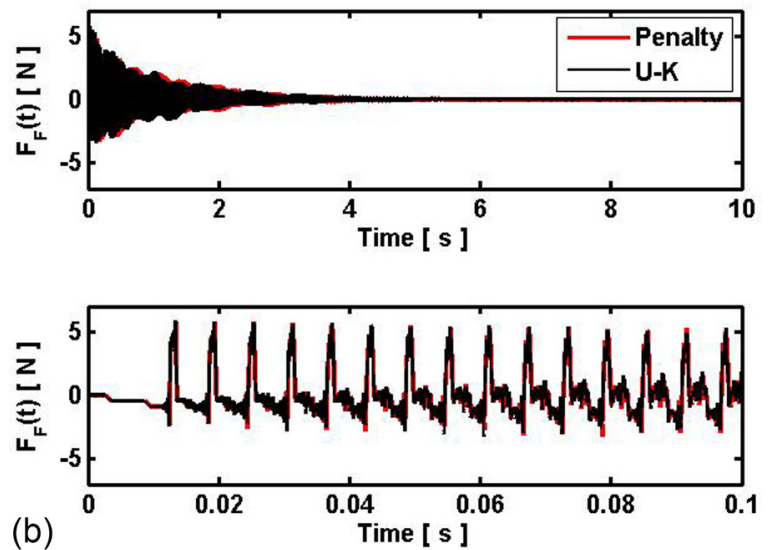

FIG. 14. (Color online) Comparison of the coupling forces computed using the U-K implementation (black) and the penalty-based formulation (red) for a string excited at $x_{E}=0.9 \mathrm{~L}$ and stopped at $x_{F}=0.33 \mathrm{~L}$ (shown are the total simulated response and a detail of the starting transient): Coupling force at the flexible bridge (top) and coupling force at the fingerboard (bottom; 150 string modes; 16 body modes).

We demonstrate that the results obtained are consistent with those previously obtained by the authors when modeling string/body coupled vibrations using penalty methods for the constraints. However, numerically, the present approach proved significantly more efficient. It is currently being extended to address intermittent constraints between subsystems.

\section{ACKNOWLEDGMENTS}

The authors acknowledge the Fundação para Ciências e Tecnologia (FCT) for the financial support of C2TN and INET-md through the Projects UID/Multi/04349/2013 and UID/EAT/00472/2013, respectively. The authors also thank the anonymous reviewers for their constructive comments, and Fabrice Galzin for lending the classical guitar.

\section{APPENDIX}

For time simulations, the U-K formulation is implemented using a velocity-Verlet algorithm. For each timestep, $h=t_{i+1}-t_{i}$, the implementation is as follows:
(1) From the solution at time $t_{i}$ the modal displacements and velocities are estimated, respectively, at time $t_{i+1}$ and the half-step $t_{i+1 / 2}$

$$
\begin{aligned}
& \boldsymbol{q}\left(t_{i+1}\right)=\boldsymbol{q}\left(t_{i}\right)+h \dot{\boldsymbol{q}}\left(t_{i}\right)+0.5 h^{2} \ddot{\boldsymbol{q}}\left(t_{i}\right), \\
& \dot{\boldsymbol{q}}\left(t_{i+1 / 2}\right)=\dot{\boldsymbol{q}}\left(t_{i}\right)+0.5 h \ddot{\boldsymbol{q}}\left(t_{i}\right) .
\end{aligned}
$$

(2) The external (non-constraining) modal forces are computed as

$$
\boldsymbol{F}\left(t_{i+1}\right)=-\boldsymbol{C} \dot{\boldsymbol{q}}\left(t_{i+1 / 2}\right)-\boldsymbol{K} \boldsymbol{q}\left(t_{i+1}\right)+\boldsymbol{F}_{\mathrm{ext}}\left(t_{i+1}\right) .
$$

(3) The modal accelerations of the unconstrained system are computed as

$$
\ddot{\boldsymbol{q}}_{u}\left(t_{i+1}\right)=\boldsymbol{M}^{-1} \boldsymbol{F}\left(t_{i+1}\right) .
$$

(4) The modal accelerations of the constrained system are computed as

$$
\ddot{\boldsymbol{q}}\left(t_{i+1}\right)=\boldsymbol{W} \ddot{\boldsymbol{q}}_{u}\left(t_{i+1}\right) .
$$

(5) The modal velocities are updated at time $t_{i+1}$ as

$$
\dot{\boldsymbol{q}}\left(t_{i+1}\right)=\dot{\boldsymbol{q}}\left(t_{i}\right)+0.5 h\left(\ddot{\boldsymbol{q}}\left(t_{i}\right)+\ddot{\boldsymbol{q}}\left(t_{i+1}\right)\right) .
$$

Allemang, R. J., and Brown, D. L. (1994). "Experimental modal analysis," in Handbook on Experimental Mechanics, edited by A. S. Kobayashi (Prentice-Hall, New York).

Arabyan, A., and Wu, F. (1998). "An improved formulation for constrained mechanical systems," Multibody Syst. Dyn. 2, 49-69.

Blajer, W. (2002). "Elimination of constraint violation and accuracy aspects in numerical simulation of multibody systems," Multibody Syst. Dyn. 7, $265-284$.

Braun, D. J., and Goldfarb, M. (2009). "Eliminating constraint drift in the numerical simulation of constrained dynamical systems," Comput. Methods Appl. Mech. Eng. 198, 3151-3160.

Debut, V., and Antunes, J. (2015). "Modelling string sympathetic vibration, string/body coupling and nonlinear string phenomena in guitars," in Proceedings of the 22nd International Congress on Sound and Vibration (ICSV22), 12-16 July 2015, Florence, Italy, pp. 2617-2624.

Debut, V., Antunes, J., Marques, M., and Carvalho, M. (2016). "Physicsbased modeling techniques of a twelve-string Portuguese guitar: A nonlinear time-domain computational approach for the multiple strings/ bridge/soundboard coupled dynamics," Appl. Acoust. 108, 3-18.

Debut, V., Carvalho, M., Marques, M., and Antunes, J. (2014). "Threedimensional time-domain simulations of the string/soundboard coupled dynamics for a twelve-string Portuguese guitar," in Congrès Français d'Acoustique (CFA 2014), 22-25 April 2014, Poitiers, France, pp. 1097-1103.

Derveaux, G., Chaigne, A., Joly, P., and Bécache, E. (2003). "Time-domain simulation of a guitar: Model and method," J. Acoust. Soc. Am. 114(6), 3368-3383.

Golub, G. H., and Van Loan, C. F. (1996). Matrix Computations (The John Hopkins University Press, Baltimore, MD), pp. 1-694.

Hemami, H., and Weimer, F. C. (1981). "Modeling of nonholonomic dynamic systems with applications," J. Appl. Mech. 48, 177-182.

Johansson, R. (1993). System Modeling and Identification (Prentice-Hall, Harlow, UK), pp. 1-528.

Laulusa, A. Bauchau, O. A. (2008). "Review of classical approaches for constraint enforcement in multibody systems," J. Comput. Nonlinear Dyn. 3(1), 011004.

Lötstedt, P. (1982). "Mechanical systems of rigid bodies subjected to unilateral constraints," SIAM J. Appl. Math. 42(2), 281-296.

Marques, M., Antunes, J., and Debut, V. (2013). "Coupled modes and timedomain simulations of a twelve-string guitar with a movable bridge," in 
Stockholm Music Acoustics Conference (SMAC 2013), edited by R. Bresin and A. Askenfeldt, 30 July-3 August 2013, Stockholm, Sweden, pp. 633-640.

Pennestri, E., Valentini, P. P., and de Falco, D. (2010). "An application of the Udwadia-Kalaba dynamic formulation to flexible multibody systems," J. Franklin Inst. 347, 173-194.

Press, W. H., Teukolsky, S. A., Vetterling, W. T., and Flannery, B. P. (2007). Numerical Recipes: The Art of Scientific Computing (Cambridge University Press, New York), pp. 1-1256.

Shabana, A. A. (2010). Computational Dynamics (Wiley, Chichester, UK), pp. 1-542.

Udwadia, F. E. (1996). "Equations of motion for mechanical systems: A unified approach," Int. J. Nonlinear Mech. 31(6), 951-958.

Udwadia, F. E., and Kalaba, R. E. (1992). "A new perspective on constrained motion," Proc. R. Soc. London A 439, 407-410.
Udwadia, F. E., and Kalaba, R. E. (1996). Analytical Dynamics-A New Approach (Cambridge University Press, New York), pp. 1-276.

Udwadia, F. E., and Kalaba, R. E. (2000). "Nonideal constraints and Lagrangian dynamics," J. Aerospace Eng. 13, 17-22.

Udwadia, F. E., and Phohomsiri, P. (2006). "Explicit equations of motion for constrained mechanical systems with singular mass matrices and applications to multi-body dynamics," Proc. R. Soc. London A 462, 2097-2117.

Woodhouse, J. (2004a). "On the synthesis of guitar plucks," Acta Acust. associated Ac. 90, 928-944.

Woodhouse, J. (2004b). "Plucked guitar transients: Comparison of measurements and synthesis," Acta Acust. associated Ac. 90, 945-965.

Yoon, S., Howe, R. M., and Greenwood, D. T. (1994). "Geometric elimination of constraint violations in numerical simulation of Lagrangian equations," J. Mech. Design 116, 1058-1064. 